ARTICLE

\title{
Malaria parasites both repress host CXCL10 and use it as a cue for growth acceleration
}

Yifat Ofir-Birin (1) 1, Hila Ben Ami Pilo', Abel Cruz Camacho', Ariel Rudik1, Anna Rivkin¹, Or-Yam Revach1, Netta Nir ${ }^{1}$, Tal Block Tamin 1 , Paula Abou Karam¹, Edo Kiper1, Yoav Peleg${ }^{2}$, Reinat Nevo ${ }^{1}$, Aryeh Solomon ${ }^{3}$, Tal Havkin-Solomon', Alicia Rojas', Ron Rotkopf (10 4, Ziv Porat (10 5, Dror Avni6,7, Eli Schwartz ${ }^{6,7}$,

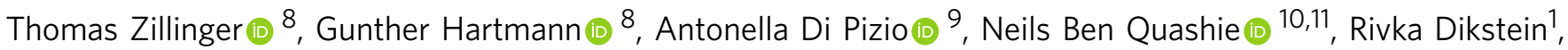
Motti Gerlic ${ }^{12}$, Ana Claudia Torrecilhas ${ }^{13}{ }^{13}$, Carmit Levy ${ }^{14}$, Esther N. M. Nolte-'t Hoen ${ }^{15}$, Andrew G. Bowie (i] ${ }^{16} \&$ Neta Regev-Rudzki (i) ${ }^{1 凶}$

Pathogens are thought to use host molecular cues to control when to initiate life-cycle transitions, but these signals are mostly unknown, particularly for the parasitic disease malaria caused by Plasmodium falciparum. The chemokine CXCL10 is present at high levels in fatal cases of cerebral malaria patients, but is reduced in patients who survive and do not have complications. Here we show a Pf 'decision-sensing-system' controlled by CXCL10 concentration. High CXCL10 expression prompts $P$. falciparum to initiate a survival strategy via growth acceleration. Remarkably, $P$. falciparum inhibits CXCL10 synthesis in monocytes by disrupting the association of host ribosomes with CXCL10 transcripts. The underlying inhibition cascade involves RNA cargo delivery into monocytes that triggers RIG-I, which leads to HUR1 binding to an AU-rich domain of the CXCL10 3'UTR. These data indicate that when the parasite can no longer keep CXCL10 at low levels, it can exploit the chemokine as a cue to shift tactics and escape.

\footnotetext{
${ }^{1}$ Faculty of Biochemistry, Department of Biomolecular Sciences, Weizmann Institute of Science, Rehovot, Israel. ${ }^{2}$ Structural Proteomics Unit, Department of Life Sciences Core Facilities (LSCF), Weizmann Institute of Science, Rehovot, Israel. ${ }^{3}$ Department of Biological Regulation, Weizmann Institute of Science, Rehovot, Israel. ${ }^{4}$ Department of Life Sciences Core Facilities, Weizmann Institute of Science, Rehovot, Israel. ${ }^{5}$ Flow Cytometry Unit, Life Sciences Core Facilities, Weizmann Institute of Science, Rehovot, Israel. ${ }^{6}$ The Institute of Geographic Medicine and Tropical Diseases and the Laboratory for Tropical Diseases Research, Sheba Medical Center, Ramat Gan, Israel. ${ }^{7}$ Faculty of Medicine, Sackler School of Medicine, Tel Aviv University, Tel Aviv, Israel. ${ }^{8}$ Institute of Clinical Chemistry and Clinical Pharmacology, University Hospital Bonn, Bonn, Germany. ${ }^{9}$ Leibniz-Institute for Food Systems Biology at the Technical University of Munich, Technical University of Munich, Freising, Germany. ${ }^{10}$ Epidemiology Department, Noguchi Memorial Institute for Medical Research, College of Health Sciences, University of Ghana, Legon, Ghana. ${ }^{11}$ Centre for Tropical Pharmacology and Therapeutics, University of Ghana Medical School, Accra, Ghana. ${ }^{12}$ Department of Clinical Microbiology and Immunology, Sackler Faculty of Medicine, Tel Aviv University, Tel Aviv, Israel. ${ }^{13}$ Department of Pharmaceutical Sciences, Federal University of São Paulo, UNIFESP, Diadema, Brazil. ${ }^{14}$ Department of Human Genetics and Biochemistry, Tel Aviv University, Tel Aviv, Israel. ${ }^{15}$ Department of Biomolecular Health Sciences, Faculty of Veterinary Medicine, Utrecht University, Utrecht, The Netherlands. ${ }^{16}$ School of Biochemistry and Immunology, Trinity Biomedical Sciences Institute, Trinity College Dublin, Dublin, Ireland.

凶email: neta.regev-rudzki@weizmann.ac.il
} 
$\mathrm{T}$ he severe parasitic disease malaria is caused by the unicellular protozoa of the Plasmodium genus, with $P$. falciparum $(P f)$ and $P$. vivax responsible for the most severe clinical cases. Several billion people - nearly half the world's population - are at risk of contracting malaria, with hundreds of millions of new infections and up to half a million deaths yearly, especially of young children under the age of five ${ }^{1}$. These statistics come at a time when there is an alarming rise in resistance to all commercially available antimalarial drugs ${ }^{2}$.

A major life-threatening complication of $P f$ infection in humans is fatal cerebral malaria (CM), a neurological syndrome characterized by convulsions, seizures, and coma ${ }^{3}$. The pathogenesis of $\mathrm{CM}$ is multi-factorial, involving reversible vascular occlusion, dysregulation of cytokine, and chemokine production and inflammation ${ }^{4-6}$. It is accepted that high parasite densities are a determinant of $\mathrm{CM}$ development ${ }^{7}$, though the mechanisms underlying CM complications are still poorly understood ${ }^{8}$.

Accumulating evidence demonstrate a prominent role for human the chemokine CXCL10 (also known as IFN- $\gamma$-inducible protein 10 [IP-10], in CM pathogenesis and mortality ${ }^{4-6}$. CXCL10 is a $\sim 10-\mathrm{kDa}$ inflammatory chemokine that binds to CXCR3 (G protein-coupled receptor 9 (GPR9), CD183) to mediate immune responses through the activation and recruitment of leukocytes, such as T cells and NK cells ${ }^{9}$. It is induced by IFN- $\gamma$ and TNF and secreted by various cell types, such as monocytes and neutrophils ${ }^{10}$. Intriguingly, malaria patient cohort studies from endemic countries have found CXCL10 to be a highly accurate biomarker for CM disease progression ${ }^{4-6}$. Out of the many chemokines and cytokines measured in patient sera, CXCL10 levels rose as disease severity increased, with the highest levels noted for fatal cases of $\mathrm{CM}^{4-6}$. Surprisingly, the level of this chemokine in early stages of the infection and in the non-fatal malaria patients is relatively low ${ }^{11}$. This finding is also supported by an analysis of a large clinical data set of $P f$-infected patients, which indicates that low $P f$ parasitemia is associated with lower CXCL10 levels ${ }^{12}$. Furthermore, CXCL10 neutralization or genetic deletion in a malaria mice model alleviates brain intravascular inflammation and protects Plasmodium berghei ANKA-infected mice from $\mathrm{CM}^{13}$. While these studies point to CXCL10 as an attractive therapeutic target, the molecular mechanism underpinning CXCL10's involvement in malaria is yet to be determined.

Malaria parasites invade human red blood cells (RBCs), rapidly developing into the ring stage, which lasts for $\sim 16 \mathrm{~h}$. Then, the parasites grow into metabolically active trophozoites, and divide into multi-nucleated schizonts, which rupture the RBC and go on to invade naïve RBCs. To ensure transmission, the parasite must differentiate into its sexual forms (gametocytes), the only forms competent for transmission to the mosquito vector (reviewed in Tadesse et al. ${ }^{14}$ )

Recent findings demonstrate that extracellular vesicles (EVs) secreted from $P f$-infected RBCs (iRBCs) have important functions in the malaria immune-evading mechanism ${ }^{15,16}$ and in disease pathogenesis ${ }^{17,}{ }^{18}$. EVs are cell-derived membrane-enclosed vesicles (e.g., microvesicles and exosomes) that carry a multitude of proteins, lipids, metabolites and nucleic acids, in which the EVs transfer to target cells providing a robust delivery system for various bioactive signals ${ }^{15,}$ 19-22 .

In malaria, the role of secreted vesicles in both parasite-parasite communication $^{23,24}$ and parasite-host interaction ${ }^{25-31}$ have been reported. Secreted EVs provide a mechanism that enables the parasite to alter host-cell responses using human RNA molecules $^{25}$ and parasitic DNA ${ }^{26}$. In essence, nucleic acids of pathogens are sensed by host pattern recognition receptors (PRRs). For example, RIG-I-like receptors sense cytosolic viral RNA, and cGAS and IFI16, which signal via STING, sense cytosolic double-stranded DNA ${ }^{32}$. Once activated, PRRs trigger signaling cascades that modify gene expression and stimulate the production of type I interferons (IFNs), chemokines and proinflammatory cytokines, which go on to activate a broad antipathogen immune response. At the same time, pathogens target these host sensors for their own benefit ${ }^{33}$. Indeed, upon monocyte internalization of parasitic DNA-harboring vesicles, a STINGdependent type I IFN and chemokine response is induced, as measured by the levels of CCL5, CXCL10, IFNA, IFNB, and IFIT1 mRNA $^{26}$.

Here we show that internalization of Pf-derived EVs into monocytes specifically restricts the translation of host CXCL10. The mechanism of CXCL10 translation inhibition is mediated by four crucial components: (I) RNA molecules that are loaded into $P f$-derived EVs; (II) Upon internalization of the EVs by monocytes, the human RNA receptor retinoic acid-inducible gene-I protein (RIG-I) is activated; (III) a small RNA domain (92 bp) enriched with AU-elements at the $3^{\prime} \mathrm{UTR}$ of the CXCL10 transcript is responsible for the translation blockage; and (IV) HUR1, a host RNA-binding protein, is recruited and directly binds to the CXCL10 transcript, thereby repressing its translation. We also provide the evidence that, in turn, CXCL10 itself promotes malaria parasite growth. Our findings point to a 'decision-sensing system' that the parasite uses to control its course of action on the basis of CXCL10 levels in the circulation. At early stages of infection, the parasite can reduce CXCL10 secretion. When the infection becomes severe (as in the case of $\mathrm{CM}$ ), CXCL10 secretion reaches high levels despite this parasitic effect. The parasite is then able to sense the heightened CXCL10, as 'an alert facilitator', prompting it to shift tactics accordingly and promotes its growth.

\section{Results}

CXCL10 protein is not secreted upon internalization of Pfderived EVs. Early (ring) stage $P f$-infected RBCs have been shown to secrete DNA-harboring EVs that are taken up by monocytes to induce a Type I IFN response ${ }^{26}$ (as opposed to EVs secreted from a later stage, Trophozoite (TR)). Internalization of parasitic DNA EV-cargo into monocytes leads to mRNA induction of the following four genes: CXCL10, CCL5, IFNA, and IFNB during the first $24 \mathrm{~h}$ post treatment (Fig. 1A). Surprisingly, when we measured the secretion level of the protein products of these genes by ELISA, only chemokine CXCL10 could not be detected (Fig. 1B). Furthermore, a Western Blot analysis demonstrated no CXCL10 protein in the cell lysate of THP-1 cells following the internalization of $P f$-derived EVs, while the protein is present upon cell transfection with the positive control, the stimulator poly(dA:dT) ${ }^{26}$ (Fig. 1C). We verified the absence of the protein within the cells also using an ELISA assay for the cell protein extract (Fig. 1D). These results indicate that parasitic EVs do not affect CXCL10 secretion, but rather abolish its expression within the cells, a lack-of-CXCL10-expression feature similar to the nontreated monocytes. Importantly, even introducing cells pretreated with $P f$-derived EVs to the stimulating poly(dA:dT) cells starkly reduced the cellular level of CXCL10, as observed by both western blot (Fig. 1E) and ELISA (Fig. 1F) analyses. We further verified the depletion of the CXCL10 protein upon internalization of the EVs by using primary monocytes obtained from three healthy donors (Fig. 1G).

We next confirmed that the absence of CXCL10 protein from monocytes is not due to a general response of monocytes to any pathogen-derived EVs by using EVs released from the human parasite Trypanosoma cruzi. Indeed, as opposed to our Pf experiments, we clearly detected the presence of CXCL10 in human monocytes following internalization of EVs derived from T. cruzi trypomastigotes (Supplementary Fig. 1). 
A.

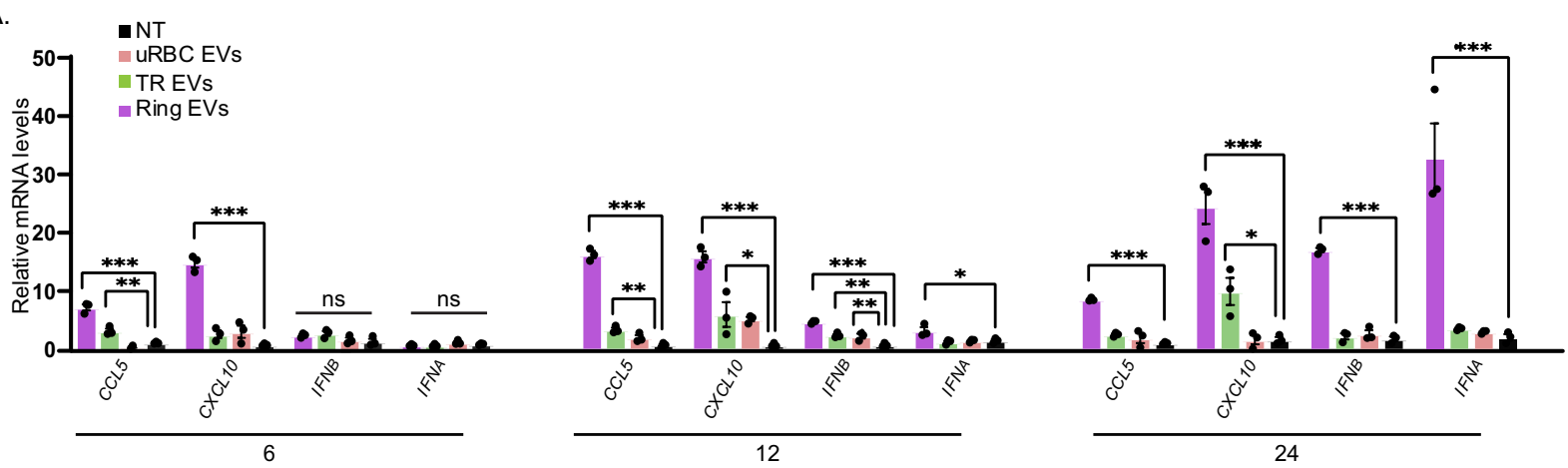

B.
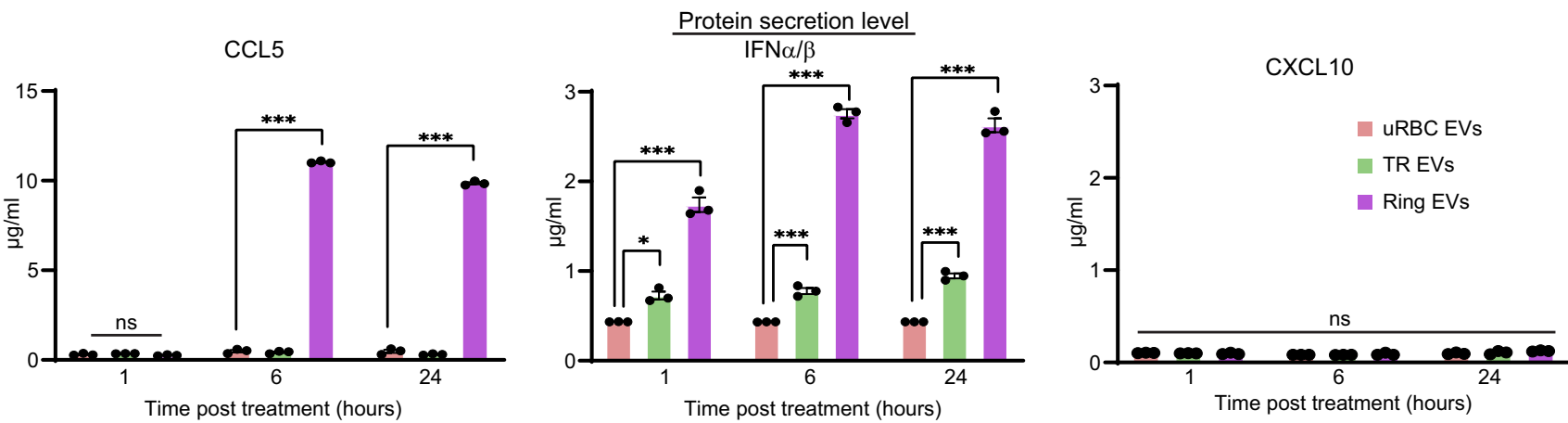

C.

D.

Cellular protein level

E.
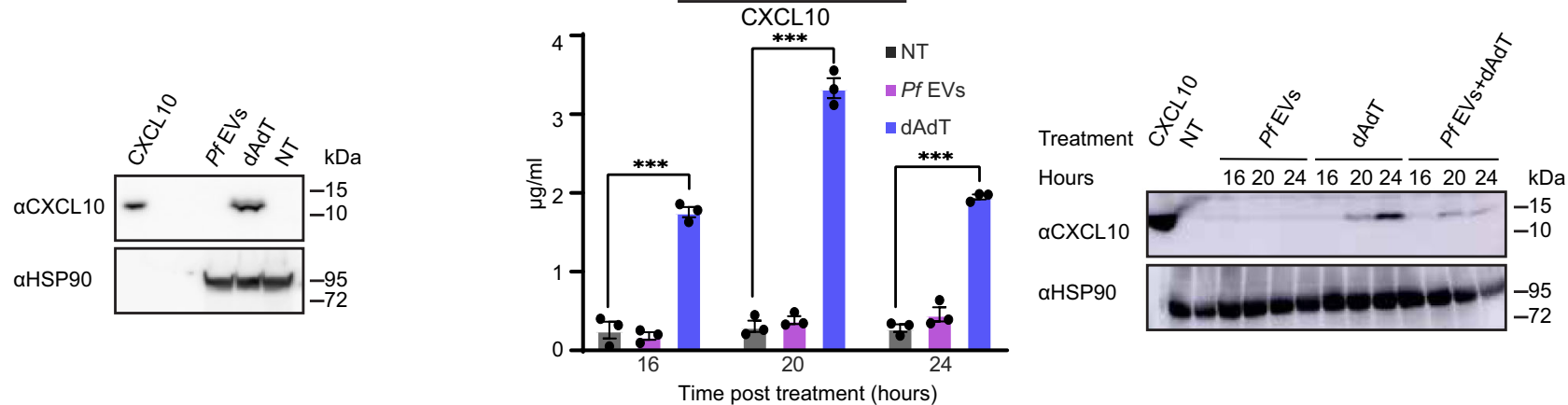

F.

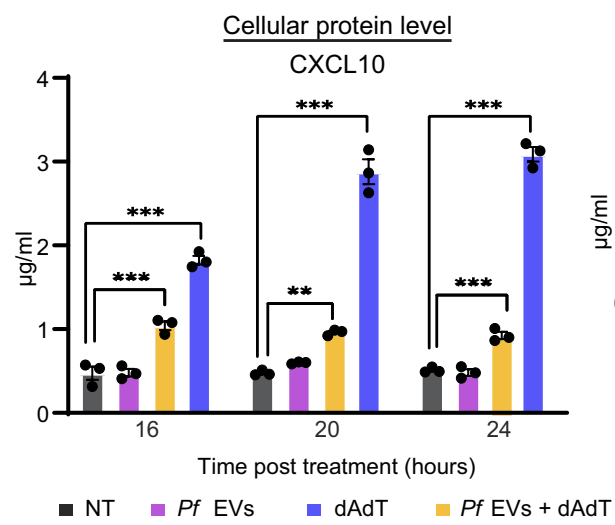

G.

Protein secretion level

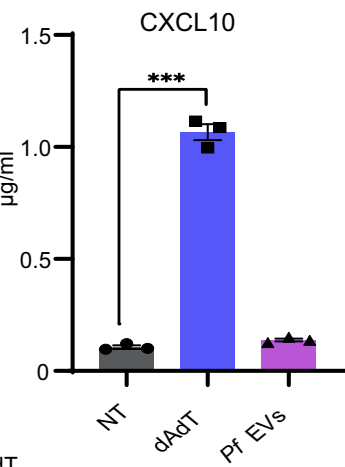

We also ruled out the possibility that the absence of CXCL10 is a result of the degradation of the protein in monocytes upon the internalization of $P f$-derived EVs. Using Western Blot analysis, we show that CXCL10 is not present in either untreated cells or cells treated with the proteasome inhibitor MG132 $2^{34}$ (anti ubiquitin was used to validate MG132 activity) (Supplementary
Fig. 2). Nor was absence of CXCL10 due to any proteolytic activity in the vesicles themselves; as shown by Western Blot analysis, we were still able to detect the purified CXCL10 following its addition to $P f$-derived EVs or EV lysate in vitro (Supplementary Fig. 3). We further examined two other known mechanisms involved in modulating protein cellular levels: (I) the 
Fig. 1 Uptake of Pf-derived EVs leads to depletion of chemokine CXCL10 from monocytes. A RT-PCR analysis of CXCL10, CCL5, IFNA, and IFNB normalized to HPRT1 of THP-1 cells treated with, trophozoite(TR)-derived EVs, ring-derived EVs or not treated (NT) for 6,12 , or $24 \mathrm{~h}$. $n=3$ biologically independent experiments, SEM, one-way ANOVA followed by Dunnett's test, (1 h- CCL5; Ring EVs - NT $P<0.001{ }^{\star \star \star}$ TR EVs - NT ${ }^{\star \star P}=0.00783$. CXCL10; Ring EVs - NT ${ }^{\star \star \star} P<0.001$. IFNA and IFNB ns not significant. 6 h-CCL5; Ring EVs - ${ }^{\star \star \star} P<0.001$, TR EVs $-N T{ }^{\star \star} P=0.00514$. CXCL10; Ring EVs $-N T{ }^{\star \star \star} P<$ 0.001 , TR EVs $-N T{ }^{\star} P=0.0338$. IFNB; Ring EVs $-N T{ }^{\star \star \star} P<0.001$, TR EVs $-N T{ }^{\star \star} P=0.00597$, uRBC EVs $-N T{ }^{\star \star} P=0.00907$. IFNA; Ring EVs $-\mathrm{NT}{ }^{\star} P=$ 0.0229. 24 h- CCL5; Ring EVs - NT ${ }^{\star \star \star} P<<0.001$. CXCL10; Ring EVs - NT ${ }^{\star \star \star} P<0.001$, TR EVs $-N T{ }^{\star} P=0.0417$. IFNB; Ring EVs $-N T{ }^{\star \star \star} P<0.001$. IFNA; Ring EVs - NT $\left.{ }^{* \star *} P<0.001\right)$. B THP-1 cells were incubated with Pf-ring-stage-, Pf-trophozoite-stage- or uRBC-derived vesicles for 1, 6, and 24 h. An ELISA assay was performed on the cells' media to detect secreted CCL5 and CXCL10. HEK blue IFN $\alpha / \beta$ assay was performed. $n=3$ biologically independent experiments, SEM, one-way ANOVA followed by Dunnett's test, CXCL10; ns. CCL5; 1-h-ns, 6 h- Ring EVs - uRBC EVs ${ }^{\star \star *} P<1 e-10,24$ h- Ring EVs - uRBC $\mathrm{EVs}{ }^{\star \star \star} P=1.03 \mathrm{e}-10$. IFN $\alpha / \beta-1 \mathrm{~h}$ - Ring EVs $-\mathrm{uRBC}{ }^{\star \star \star} P=4.17 \mathrm{e}-06, \mathrm{TR} \mathrm{EVs}-\mathrm{uRBC}$ EVs ${ }^{\star} P=0.0138 .6 \mathrm{~h}-$ Ring EVs $-\mathrm{uRBC}$ EVs ${ }^{\star \star \star} P=1.24 \mathrm{e}-08, \mathrm{TR}$ EVs uRBC EVs ${ }^{\star \star \star} P=0.000847,24 \mathrm{~h}$ - Ring EVs - uRBC EVs ${ }^{\star \star \star} P=9.66 \mathrm{e}-08$, TR EVs - uRBC EVs $\left.{ }^{\star \star \star} P=0.000459\right)$. C Western Blot assay of CXCL10 and HSP90 (loading control) was performed on THP-1 cell lysate. THP-1 cells were incubated with Pf-ring-stage-derived EVs and then transfected with poly(dA: dT) or not treated (NT) for $16 \mathrm{~h}$ before harvesting. Results are representative of at least three independent biological replicates. D THP-1 cells were incubated with Pf-ring-stage-derived EVs and then transfected with poly(dA:dT) or NT for 16, 20 or $24 \mathrm{~h}$. An ELISA assay was performed on the cell lysate

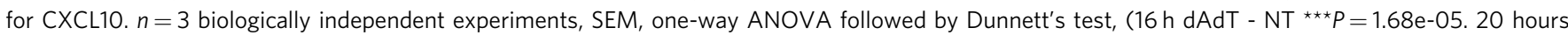
$\mathrm{dAdT}-\mathrm{NT}{ }^{\star \star \star} P=6.28 \mathrm{e}-07.24 \mathrm{~h}$ dAdT $\left.-\mathrm{NT}{ }^{\star \star \star} P=2.44 \mathrm{e}-06\right)$. E Western Blot analysis of THP-1 cell lysate. THP-1 cells were incubated with Pf-ring-stagederived EVs, transfected with poly $(\mathrm{dA}: \mathrm{dT})$ and treated with $P$ f-ring-stage-derived EVs for $1 \mathrm{~h}$ before being transfected with poly(dA:dT). The cells were harvested 16, 20, and $24 \mathrm{~h}$ post treatment. Antibodies were used against CXCL10 and HSP90 (loading control). NT not treated. Results are representative of at least three independent biological replicates. F ELISA assay for CXCL10 was performed on cell lysate. THP-1 cells were incubated with Pf-ring-stagederived EVs, transfected with poly $(\mathrm{dA}: \mathrm{dT})$, and then treated with Pf-ring-stage-derived EVs for $1 \mathrm{~h}$ before being transfected with poly $(\mathrm{dA}: \mathrm{dT})$. Cells were harvested 16,20 , and $24 \mathrm{~h}$ post treatment NT-not treated. $n=3$ biologically independent experiments, SEM. One-way ANOVA followed by Dunnett's test, (16 h- dAdT - NT ${ }^{\star \star \star} P<1 \mathrm{e}-04, P f \mathrm{EVs}+\mathrm{dAdT}-\mathrm{NT}{ }^{\star \star \star} P=0.000245 .20 \mathrm{~h}-\mathrm{dAdT}-\mathrm{NT}{ }^{\star \star \star} P<0.001, P f \mathrm{EVs}+\mathrm{dAdT}-\mathrm{NT}{ }^{\star \star} P=0.00475 .24 \mathrm{~h}-\mathrm{dAdT}-\mathrm{NT}$ ${ }^{\star \star \star} P<0.001, P f$ EVs + dAdT - NT $\left.P<0.001^{\star \star \star}\right)$. G ELISA assay for detection of CXCL10 in PBMC-derived monocytes. CD14+ primary cells (monocytes) isolated from three naive healthy donors. The cells were incubated with Pf-ring-stage-derived EVs or transfected with poly (dA:dT) and then analyzed by

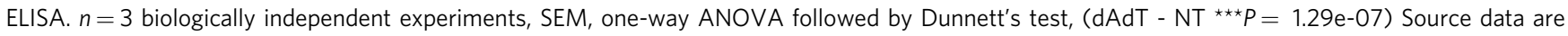
provided as a Source Data file.

stability of the CXCL10 mRNA transcripts in the presence of Pfderived EVs as compared to the positive control (Supplementary Fig. 4); and (II) nuclear-retention of the $\mathrm{mRNA}^{35}$, to test whether the EVs are responsible for blocking the export of CXCL10 transcripts from the nucleus to the cytosol, by measuring the maturation rate of the CXCL10 transcript within the nucleus (Supplementary Fig. 5). In both cases, the EV-treated cells behaved similarly to the positive control, indicating that CXCL10 mRNA is relatively stable and gets exported into the cytosol. Hence, none of these mechanisms is responsible for the cellular absence of CXCL10 in EV-treated monocytes.

Pf-derived EVs inhibit the translation of CXCL10 by disrupting the host ribosome. Next, we directed efforts to address whether the internalization of the EV cargo prevents the expression of CXCL10 via an active process of interfering with the ribosome's association to the CXCL10 transcript. This was determined by assessing the cellular distribution of CXCL10 mRNA in polyribosomes using polysome profiling. The UV absorbance spectra (OD254) of the polysome sucrose gradients demonstrated a similar ribosome distribution profile between the EV-treated and non-treated cells (Fig. 2A), indicating that the parasitic EVs do not globally regulate the translation status of the recipient monocytes.

CXCL10 mRNA level was quantified in each fraction of the polysome sucrose gradient using a $\mathrm{qPCR}$ assay. Remarkably, in the presence of the parasitic EVs, the majority of CXCL10 mRNAs shifted to the free RNA fraction, in contrast to its accumulation in the polysome fraction isolated from control cells (treated with the stimulator poly(dA:dT)), where the majority of CXCL10 transcripts were detected within the heavily translated fraction (Fig. 2B). IFNB mRNAs (used as control for Type Irelated transcripts) were found in both samples mostly in the heavy polysome fraction (Fig. 2C). These findings show that, as a result of the internalization of the parasitic EVs, a significantly larger proportion of CXCL10 mRNAs is not associated with the host ribosomes, indicating that the synthesis of this protein is interrupted at the translation step.

Exclusive RNA molecules regulate CXCL10 translation. The activation of the CXCL10 transcript is mediated by the EV-DNA cargo via STING ${ }^{26}$ and cGAS activation, which was confirmed also by using cGAS KO THP-1 cells (Supplementary Fig. 6). In order to find the EV cargo component involved in the translation inhibition mechanism of CXCL10, we established a system to test the effect of the EV-RNA cargo on CXCL10 translation. Specifically, we questioned whether a single parasitic-sourced RNA or rather a distinct collection of RNA molecules facilitates the translation inhibition of CXCL10. To address this, we set up an experimental system where CXCL10 transcripts were initially upregulated (by transfecting $P f$ genomic DNA (gDNA) to THP- 1 cells, known to induce CXCL10 expression ${ }^{26}$ ) within monocytes. We then examined CXCL10 protein expression levels following the introduction of RNA derived from different parasitic origins. We harvested total RNA from (I) Ring or trophozoite parasite cells, and (II) EVs secreted from ring or trophozoite parasite cultures (Fig. 3A). We tested the individual effect of each of these four distinct RNA samples on CXCL10 secretion (Fig. 3B) and on CCL5 secretion as control (Supplementary Fig. 7). The RNA was transfected to monocytes using Lipofectamine RNAiMAX reagent $^{36}$ and CXCL10 secretion was then measured by ELISA (Fig. 3B). The RNA transfection was confirmed using a PCR reaction targeted to the ETRAMP 11.2 gene, an EV parasitic RNA marker $^{26}$ (Supplementary Fig. 8). We initially verified that the transfection of the EV-RNA cargo does not upregulate CXCL10 transcription levels (Supplementary Fig. 9). Monocytes stimulated with transfected Pf DNA served as a positive control for CXCL10 secretion. Remarkably, we found that only the RNA harvested from EVs secreted from the ring (early) stage significantly reduced the secretion level of CXCL10, similar to the non-treated cells, while none of the other three RNA-sources did; in the presence of each of the other RNA samples, CXCL10 secretion levels were high (Fig. 3A). Since the parasitic 
A.

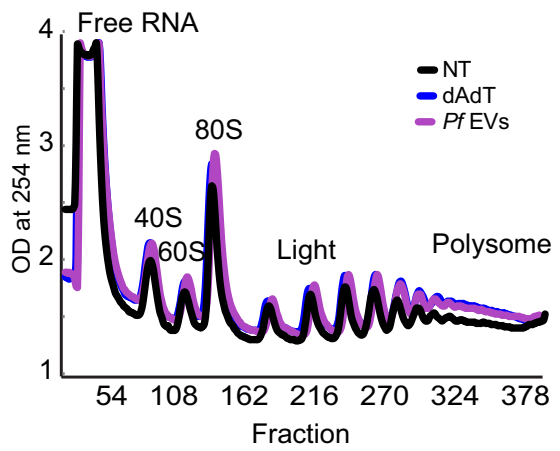

B.

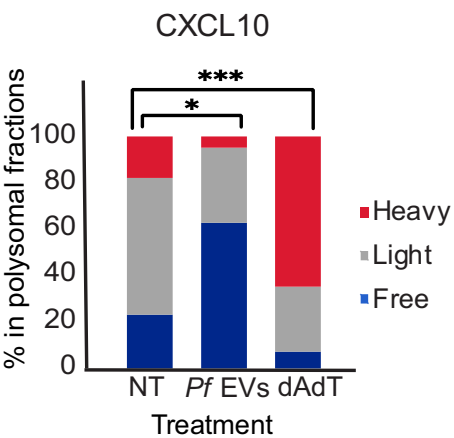

C.

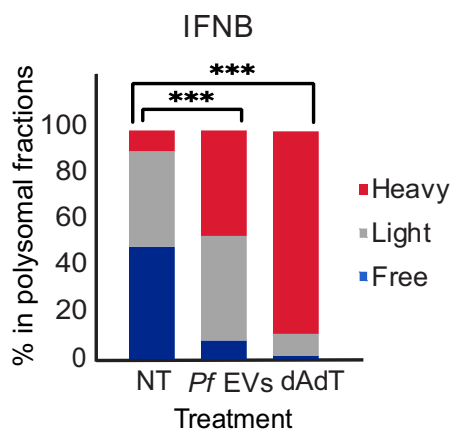

Fig. 2 Pf-derived EVs inhibit the translation of CXCL10. A Polysomal profiling of THP-1 cells $14 \mathrm{~h}$ post transfection with poly(dA:dT) (blue), Pf -derived EVs (purple), or not treated (NT) (black). One representative repeat out of five biological replicates is shown. B. THP-1 cells were incubated with Pf-ringstage-derived EVs or transfected with poly(dA:dT) for $14 \mathrm{~h}$ or NT. Polysomal profiling was performed on all samples and RNA was isolated from heavy, light and free fractions. Real-time PCR for CXCL10 was performed on all the polysome fractions. $n=3$ biologically independent experiments, NT (not treated) THP-1 cells were used as control. One-way ANOVA followed by Dunnett's test for the heavy fractions (dAdT - NT ${ }^{\star \star \star} P=1.64 \mathrm{e}-05, P f \mathrm{EV} s-\mathrm{NT}{ }^{\star} P$ $=0.0368)$. C. THP-1 cells were incubated with Pf-ring-stage-derived EVs, transfected with poly(dA:dT) for $14 \mathrm{~h}$ or NT. Ribosomal profiling was performed on all samples and RNA was isolated from heavy, light and free fractions. Real-time PCR for IFNB was performed on all the polysome fractions. $n=3$

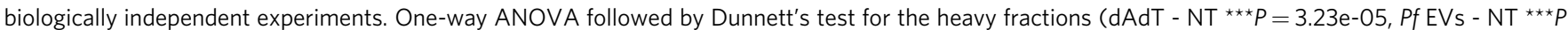
$=0.000543$ ). Source data are provided as a Source Data file.

DNA within the EVs is needed to induce CXCL10 transcription ${ }^{26}$, as we expected, when monocytes were transfected with parasitic RNA only, i.e., were not subjected to gDNA treatment, no activation was detected for CXCL10 secretion, verifying that the RNA species themselves are not sufficient to induce the immune response (Fig. 3B) and that the DNA cargo is essential, as previously reported ${ }^{26}$. These results indicate that not every RNA molecule of parasitic origin leads to the inhibition of CXCL10 translation but, rather, there is a unique RNA subpopulation that, most probably, is highly enriched and sorted into the EVs the parasite secretes at the early stage of the blood stage, i.e., the first $16 \mathrm{~h}$ post-invasion into its host $\mathrm{RBC}$, but not later on. The spectrum of RNA types implicated in this regulation may include single-stranded (ss) RNA, double-stranded (ds) RNA and, due to the presence of DNA, also RNA-DNA hybrids.

To address whether one or more of the EV's RNA subtypes is involved in CXCL10 translation inhibition, we digested the RNA cargo purified from the vesicles using either RNase $\mathrm{H}$ enzyme, for degrading DNA-RNA hybrids, or RNase I enzyme, for degrading ssRNA molecules. We found that both digestion assays, RNase $\mathrm{H}$ and RNase I, showed partial rescue of the CXCL10 translation inhibition phenotype (Fig. 3C). Combining RNase H and RNase I treatments also showed rescue of the CXCL10 translation inhibition phenotype (Supplementary Fig. 10), suggesting that both RNA-DNA hybrids and ssRNA might be involved in this regulation.

RIG-I is required for CXCL10 translation inhibition. Having established that the RNA cargo loaded within Pf-derived EVs plays a key role in CXCL10 translation regulation, we directed our efforts to identify host proteins that are essential for this regulation. In particular, we searched for candidates of the immune RNA sensor family that can sense the parasitic RNA, on the one hand, and be involved in the mechanism of translation regulation, on the other hand. Retinoic acid-inducible gene-I protein (RIG-I) is a major intracellular immune sensor of pathogenic RNA. This protein detects foreign RNA and subsequently induces a type I IFN response ${ }^{37}$. Moreover, previous work on RNA viruses has demonstrated that STING is required to restrict the translation of viral and host mRNAs, and that this translation inhibition is dependent on RIG-I's function ${ }^{38}$. Since we have already shown that STING is activated in our system ${ }^{26}$, these findings prompted an examination of RIG-I's role in CXCL10 translation inhibition in response to $P f$-derived EVs. To address the involvement of RIG-I, we used RIG-I knockout (KO) THP-1 cells generated by the CRISPR/Cas9 system. CXCL10 secretion induction was examined using both ELISA and Western Blot analyses upon EV intake by monocytes. Compellingly, while control wild-type ( $w t)$ monocytes displayed robust translation inhibition for CXCL10 (Fig. 3D, E), this response was completely absent in RIG-I KO monocytes, and the chemokine was 're-expressed' and secreted, as determined by an ELISA assay (Fig. 3D). The phenotype could be rescued by transfecting RIG-I KO cells with a HA-RIG-I plasmid (Supplementary Fig. 11). These results demonstrate that RIG-I plays a key role in the cascade that regulates CXCL10 protein synthesis, achieved by sensing either the ssRNA cargo or the RNA-DNA hybrid cargo of the parasitic vesicles.

Furthermore, as there are at least two known pathways for RIG-I activation, a conventional ${ }^{39}$ and non-conventional one ${ }^{38}$, we examined whether this RIG-I stimulation cascade is controlled through MAVS (mitochondrial antiviral signaling protein) conventional downstream activation, a stimulator of IFN genes ${ }^{37}$. This was achieved using MAVS KO THP-1 cells generated by the CRISPR/Cas9 system. We found that in MAVS KO monocytes treated with $P f$-derived EVs, similar to in the control $w t$ monocytes, CXCL10 translation is still diminished (Supplementary Fig. 12) despite the higher CXCL10- mRNA levels (Supplementary Fig. 13). These results indicate that the signaltransducing activity of RIG-I in response to the EV RNA cargo is not via MAVS but rather via a different pathway, most probably one similar to the previously reported translation inhibition mechanism $^{38}$. Moreover, transfecting THP-1 cells with the RIG-I known activator $5^{\prime}$ triphosphate dsRNA ${ }^{40}$ with or without $P f$ DNA led to the secretion of CXCL10 (Supplementary Fig. 14). This observation strengthens the claim that RIG-I activation is via a non-conventional pathway associated with CXCL10 translation inhibition.

An AU-rich domain in CXCL10 transcript facilitates the translation inhibition. Several modes of translation inhibition 
A.

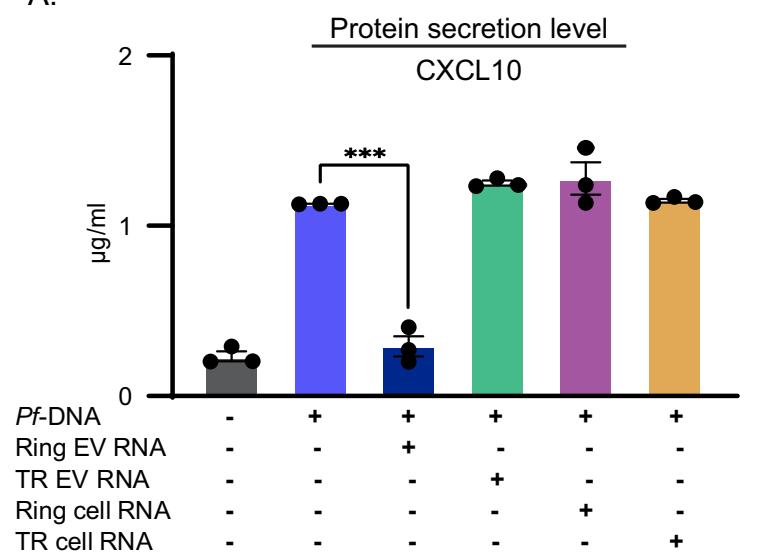

C.

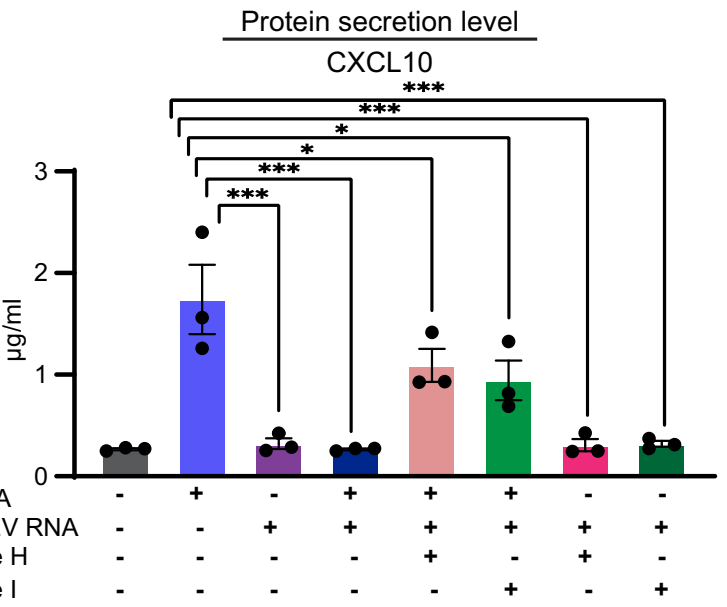

B.
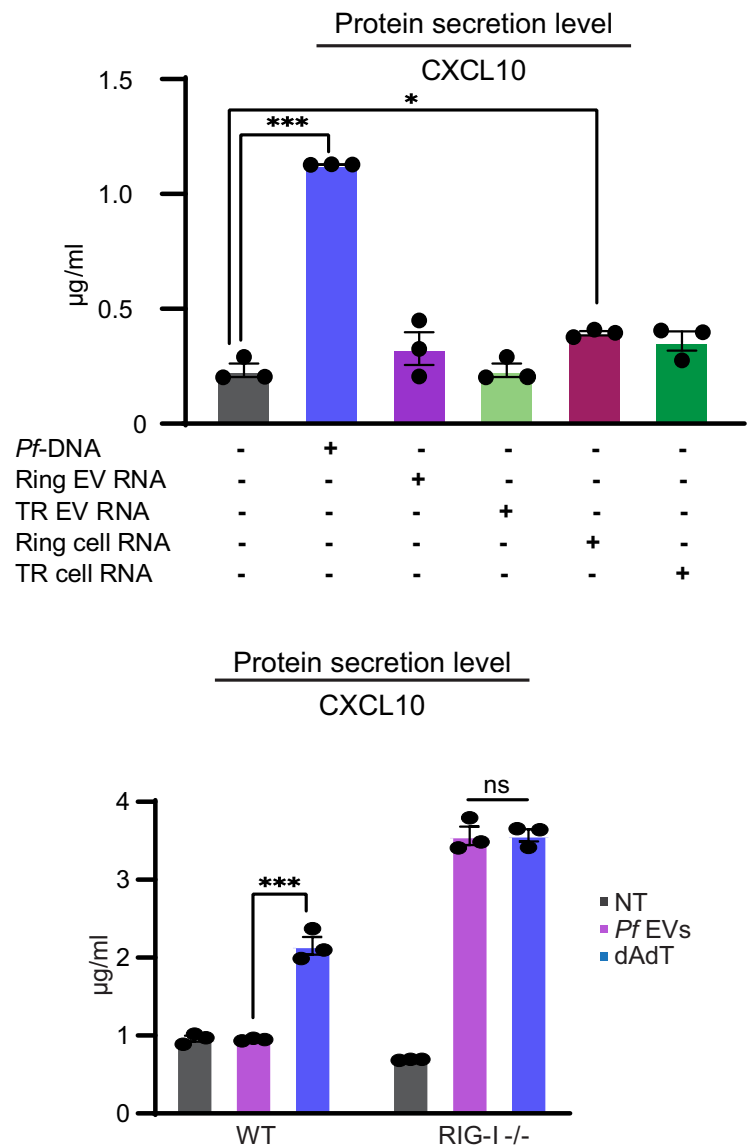

E.

THP1 RIG-I KO

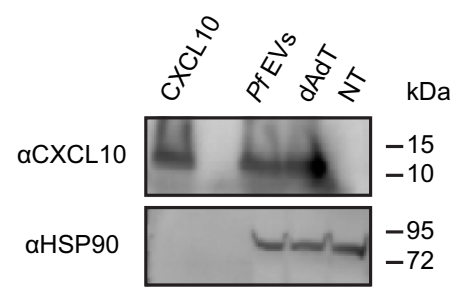

Fig. 3 Selected RNA cargo mediates CXCL10 translation inhibition via the RIG-I receptor. A THP-1 cells were transfected with RNA purified from: Pf ring stage, trophozoite stage, Pf-ring-stage-derived EVs or EVs derived from Pf-trophozoite-(TR) stage. In addition, all the cells were transfected with Pf gDNA. An ELISA was performed on the cell media for CXCL10 $16 \mathrm{~h}$ post transfection. $n=3$ biologically independent experiments, SEM, one-way ANOVA followed by Dunnett's test (Pf-DNA + Ring EVs RNA - Pf-DNA ${ }^{\star \star \star} P<0.001$ ). NT not treated. B THP-1 cells were transfected with Pf gDNA, NT, or RNA purified from: Pf ring stage, trophozoite stage, Pf-ring-stage-derived EVs, or Pf-trophozoite-stage-derived EVs. An ELISA was performed on the cell media for

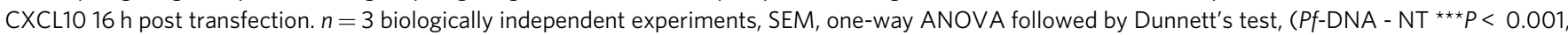
Ring-cell RNA - NT $\left.{ }^{*} P=0.0405\right)$. C THP-1 cells were transfected with RNA purified from: Pf-derived EVs, Pf-derived-EVs pretreated with RNase H and Pfderived EVs pretreated with RNase I. In addition, the cells were transfected with Pf gDNA or NT. An ELISA was performed on the cell media for CXCL10 $16 \mathrm{~h}$ post transfection. $n=3$ biologically independent experiments, SEM, one-way ANOVA followed by Dunnett's test, (NT-Pf-DNA ***P<0.001, Pf-DNA

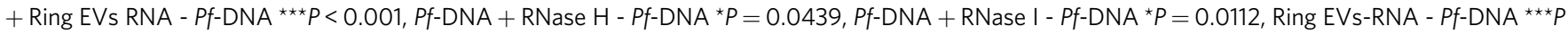
$<0.001$, RNase H - Pf-DNA ${ }^{\star \star *} P<0.001$, RNase I - Pf-DNA ${ }^{\star \star *} P<0.001$ ). D THP-1 RIG-I KO cells were incubated with EVs harvested from ring-stage Pf (iRBCs) and then transfected with poly(dA:dT) or NT for $24 \mathrm{~h}$. An ELISA assay for detecting CXCL10 was performed on cell lysates. $n=3$ biologically

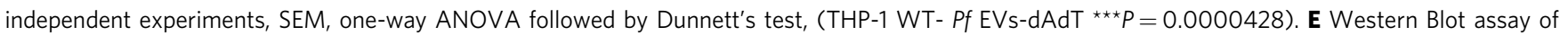
CXCL10 and HSP90 (loading control) were performed on THP-1 RIG-I KO cells. The THP-1 cells were incubated with Pf-ring-stage-derived EVs, transfected with poly (dA:dT) or not treated (NT) $16 \mathrm{~h}$ before harvesting. Results are representative of at least three independent biological replicates. Source data are provided as a Source Data file. 
are known, among them global and sequence-specific. In our case, the ribosomal profile indicates that there is no global translation inhibition within monocyte recipient cells (Fig. 2A). Thus, we focused on the sequence-specific translation inhibition mode. Sequence-specific translation inhibition is facilitated by several mechanisms (reviewed in refs. ${ }^{41,42}$ ), including cellular regulators interacting with sequences, such as the $3^{\prime}$ untranslated region $\left(3^{\prime}\right.$ UTR) of the gene, thereby marking the RNA either for degradation (reviewed in Treiber et al. ${ }^{43}$ ) or translation inhibition (reviewed in Szostak et al. ${ }^{42}$ ). Conserved AU-rich elements are known for their ability to fine-tune mRNA translation and stability ${ }^{44}$. During inflammation, the expression of various chemokine genes is regulated by their AU-rich domain, and by that, the course of inflammation is modulated ${ }^{45-47}$. Indeed, computational analysis suggests that the CXCL10 RNA sequence contains several AU-rich elements, with high abundance at the $3^{\prime}$ $\mathrm{UTR}^{48}$ (Fig. 4A).

To examine if regulatory elements at the $3^{\prime} \mathrm{UTR}$ of CXCL10 are involved in the translation inhibition, we conducted an experiment using the dual luminescence assay ${ }^{49}$, in which CXCL10 $3^{\prime}$ UTR (813 bp) was fused to a Gaussia luciferase (GLuc) reporter. The reporter gene is constitutively transcribed and translated unless there is translation regulation in this region, which would lead to a decrease in reporter luminescence. THP-1 cells were treated with $P f$-derived EVs for 5 or $16 \mathrm{~h}$ prior to the transfection with the CXCL10 3'UTR-fused-reporter. THP-1 culture media was collected and the GLuc translation levels were measured. As seen in Fig. 4B, a substantial reduction in the signal of the $3^{\prime} \mathrm{UTR}$ reporter expression was detected upon treatment with the EVs. In contrast, in the RIG-I KO cells the fused-reporter protein was highly expressed also upon treatment of the parasitic EVs (Fig. 4C). Altogether, these results demonstrate that a central element in the $3^{\prime}$ UTR sequence of CXCL10 transcript plays a role in the EV-mediated translation inhibition mechanism of the protein.

There are numerous AU-rich elements along CXCL10 3'UTR sequence ( $\sim 6$ centers of elements). In order to further dissect the area which is involved in the translation regulation, we generated three sub-domains: $\Delta \mathrm{I}(0-352 \mathrm{bp}), \Delta \mathrm{II}(357-449 \mathrm{bp})$, and $\Delta \mathrm{III}$ (455-813 bp) (Fig. 4D). Each of these deletions was fused to a luciferase reporter gene. THP-1 cells were initially treated with $P f$ derived-EVs for $16 \mathrm{~h}$ prior to the transfection with the complete CXCL10 3'UTR-fused-reporter, or each of the sub-domains $\Delta \mathrm{I}$, $\Delta \mathrm{II}$, and $\Delta \mathrm{III}$. Non-treated cells served as control. THP-1 culture media was then collected and the GLuc translation levels were measured. As seen in Fig. 4E, only for the $\Delta \mathrm{II}$ sub-domain deletion, the translational inhibition effect was lost since it was expressed under treatment with $P f$-derived EVs. This $\Delta \mathrm{II}$ subdomain significantly increased the signal of the reporter expression as compared to $\Delta \mathrm{I}$ and $\Delta \mathrm{III}$ sub-domains. Indeed, $\Delta \mathrm{II}$ sub-domain harbors the largest number of AU elements $(\sim 5$ different locations) potentially involved in the chemokine translation regulation. Activation with $5^{\prime}$ triphosphate dsRNA did not show any significant difference in luciferase activity, similarly to RIG-I KO cells (Supplementary Fig. 15).

Together, these data demonstrate that a key AU element located in the $3^{\prime} \mathrm{UTR}$ sequence of the CXCL10 transcript plays a role in the EV-mediated translation inhibition mechanism of the protein.

HUR1 binds to CXCL10 mRNA and is involved in its translation inhibition. We next sought to identify the host protein that binds CXCL10 $3^{\prime}$ UTR upon internalization of $P f$-derived EVs. As we found that AU-rich elements are essential for the transcriptional regulation of CXCL10, we reasoned that these elements are most probably involved in the inhibition regulation, and that a host RNA-binding protein directly binds to these elements. HUR1/ELAVL1 is an RNA-binding protein known to bind AU-rich elements located on the $3^{\prime} \mathrm{UTR}$ of transcripts ${ }^{50}$. This protein is involved in post-transcriptional regulation of many genes ${ }^{51}, 52$, in particularly of pro-inflammatory cytokines $^{53}, 54$. We examined whether HUR1 inhibits CXCL10 translation by introducing either $P f$-derived EVs, or poly $(\mathrm{dA}: \mathrm{dT})$ as a positive control, to monocytes whose HUR1 was silenced using a small interfering (si)RNA assay (confirmed by Western Blot); monocytes transfected with a non-targeting siRNA pool served as control (Fig. 4G). We found that CXCL10 translation inhibition was significantly rescued in HUR1 siRNA cells as CXCL10 protein could now be detected by ELISA (Fig. 4F) and confirmed Western Blot (Fig. 4G), demonstrating the involvement of host HUR1 in CXCL10 repression upon the internalization of the parasitic EVs.

To further examine whether HUR1 directly interacts with CXCL10 transcripts in vivo, we pretreated monocytes with $P f$ derived EVs or poly(dA:dT), and then subjected them to a native RNA immunoprecipitation (RIP) assay, using antibodies against HUR1 (Supplementary Fig. 16) or an antibody against HSP70 (negative control). Total RNA was extracted from the antibody pull down and then the CXCL10 transcript in each sample was subjected to a qPCR analysis (Supplementary Fig. 17). As a positive control for the $\mathrm{qPCR}$ analysis, we amplified $c-M y c$, a known RNA target of HUR $1^{55}$, whereas amplification of the CCL5 transcript served as a negative control (Fig. 4H). Strikingly, despite the presence of these transcripts in the total RNA input (Supplementary Fig. 17), extremely high levels of the CXCL10 RNA could be detected in the HUR1 pull down sample. We found that only after treating the cells with $P f$-derived EVs did the host protein HUR1 bind to CXCL10 transcripts, whereas in the presence of poly(dA:dT), CXCL10 transcripts could not be detected (Fig. $4 \mathrm{H}$ ).

Importantly, we performed qPCR analysis of the RIP-HUR1 for several genes such as IFNA, IFNB HPRT1 and IFIT1 and none of the above transcript was found attached to HUR1 (Supplementary Fig. 18). Moreover, none of the target mRNAs (TNF, KLF2 and CXCL2) known to bind HUR1 ${ }^{56-59}$ exhibits enriched association upon the introduction of the parasitic EVs (Supplementary Fig. 19).

Overall, these findings indicate that HUR1 directly binds to the CXCL10 transcript and is involved in the translation inhibition mediated by Pf-derived EVs.

Notably, a HUR1-RIP assay for RIG-I KO cells post treatment with $P f$-derived EVs did not detect any CXCL10 RNA in the HUR1 pull-down sample (Supplementary Fig. 20), suggesting that RIG-I needs to be present within the cells for the binding of HUR1 to the CXCL10 transcript. In contrast, AUF1, another known binding protein to AU-rich elements ${ }^{60}, 61$, was not associated with CXCL10 transcripts when parasitic EVs were present. BCL2 served as positive control (Supplementary Fig. 21).

CXCL10 stimulates parasite growth. Chemokines are essential for stimulating leukocyte chemotaxis and initiating inflammatory responses (reviewed in Stone et al. ${ }^{62}$ ). We show that $P f$-derived EV entry into human monocytes increases the mRNA levels of CXCL1026 (Fig. 1A) but stops the synthesis of the protein, while other cytokines and chemokines of the Type 1 IFN response are upregulated at both levels (Fig. 1). We hypothesized that this translation blockage, specific to CXCL10, may be driven by a parasite mechanism favorable to their survival. Support for our hypothesis comes from accumulating evidence indicating that, for yet unknown reasons, parasite density directly correlates with 
A

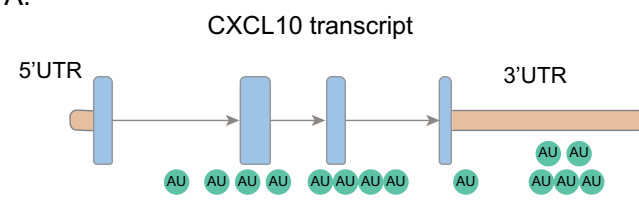

D.

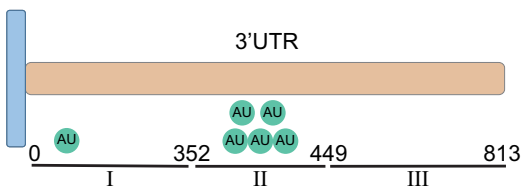

E.

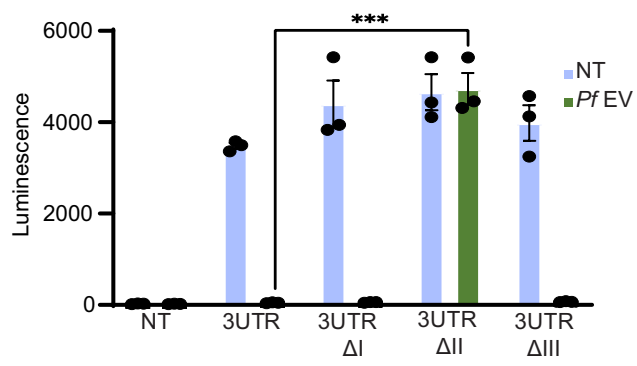

B.

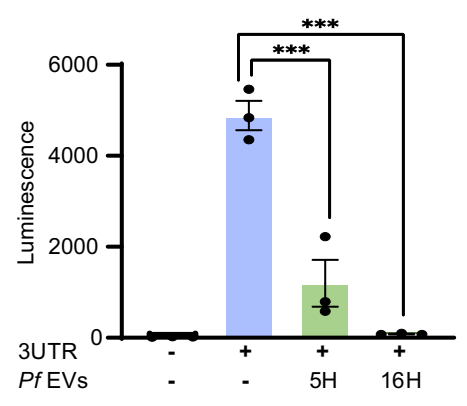

F.

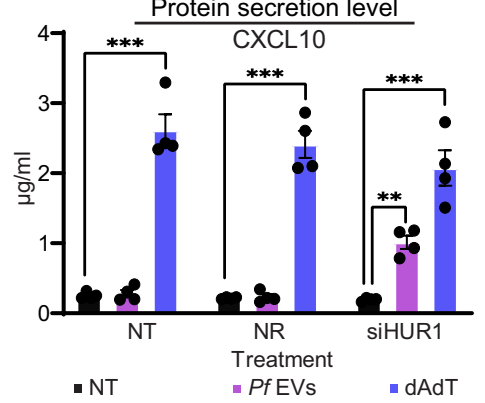

C.

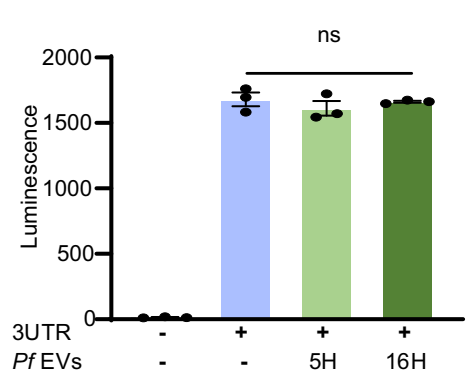

G.

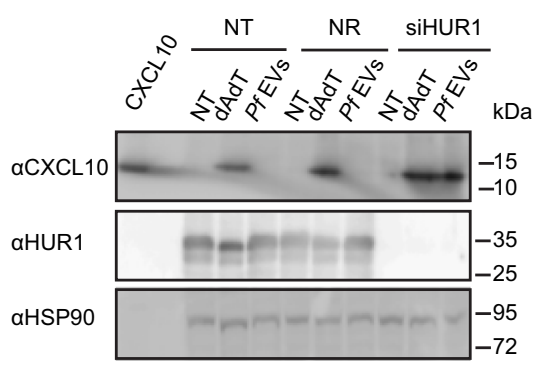

$\mathrm{H}$.

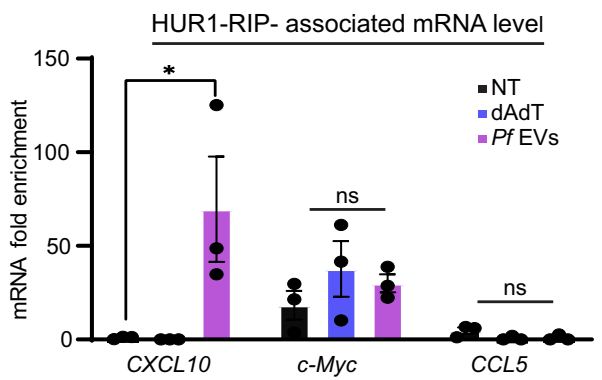

Fig. 4 HUR-1 binds to AU- rich domain in the $\mathbf{3}^{\prime}$ UTR of the CXCL10 transcript and is involved in translation inhibition. A Schematic illustration of CXCL10 mRNA. Orange squares, blue rectangles, and gray arrows represent the UTRs, exons, and introns of the CXCL10 mRNA, respectively. The green circles illustrate the AU-rich domains predicted by http://arescore.dkfz.de/ analysis. B THP-1 cells were incubated with Pf-derived EVs 5 or 16 h (H) or not treated before the transfection with the CXCL10-3'UTR-Luc plasmid. $24 \mathrm{~h}$ post transfection, the cell media was collected, luciferase levels were measured and normalized to SEAP levels. $n=3$ biologically independent experiments, SEM, one-way ANOVA followed by Dunnett's test, (3UTR+Pf EVs $16 \mathrm{~h}-{ }^{\star \star \star *} P<$ 1e-04, 3UTR + PfEVs 5 h - 3UTR ${ }^{\star \star \star} P<1$ e-04). C THP-1 RIG-I KO cells were incubated with Pf-ring-stage-derived EVs for 5 or 16 h (H) or not treated (NT) before their transfection with the CXCL10-3'UTR-Luc plasmid. 24 hours post transfection, the cell media was collected and luciferase levels were measured and normalized to the SEAP levels. $n=3$ biologically independent experiments, SEM, one-way ANOVA followed by Dunnett's test (ns). D Schematic illustration of the three sub-domains of CXCL10 $3^{\prime}$ UTR and the cloned deletions $\Delta \mathrm{l}, \Delta \mathrm{Il}$, and $\Delta \mathrm{III}$. E THP-1 cells were treated with Pf -derived EVs for $16 \mathrm{~h}$ prior to transfection with CXCL10 3'UTR-fused-reporter, $\Delta \mathrm{l}, \Delta \mathrm{ll}, \Delta \mathrm{Ill}$ plasmids (NT-not treated cells). 24 hours post-transfection the cells media was collected, Luciferase levels were measured and normalized to the SEAP levels. $n=3$ biologically independent experiments, SEM, one-way ANOVA followed by Dunnett's test, (Pf EVs- 3UTR $\Delta I I-3 U T R{ }^{\star \star \star} P<1 \mathrm{e}-06$ ). F THP-1 cells were transfected with siRNA for HUR1 or with non-targeting siRNA pool (NR). 48 hours post siRNA transfection, cells were treated with Pf-derived EVs, with poly(dA:dT) and following $24 \mathrm{~h}$ post treatments, secreted CXCL10 in the cells media was measured by an ELISA assay, $n=4$ biologically independent experiments, SEM, one-way ANOVA followed by Dunnett's test, (NT- Pf-DNA - NT ${ }^{\star \star \star} P=1.14 \mathrm{e}-06, \mathrm{NR}-\mathrm{Pf}-\mathrm{DNA}-\mathrm{NT}{ }^{\star \star \star} P=5.14 \mathrm{e}-07$, siHUR1-Pf-DNA - NT ${ }^{\star \star \star} P=2.59 \mathrm{e}-05, P f-\mathrm{EV}$ s $-\mathrm{NT}{ }^{\star \star} P=0.00907$ ). NT (not treated). G THP-1 cells were transfected with siRNA for HUR1 or with non-targeting siRNA pool (NR). 48 hours post siRNA transfection, cells were treated with Pf-derived EVs, with poly (dA:dT) and following $24 \mathrm{~h}$ post treatments, HUR1 reduction and CXCL10 translation was verified by Western Blot analysis. HSP90 used as a loading control. NT (not treated). Results are representative of at least three independent biological replicates. H THP-1 cells were treated with Pf-derived EVs or transfected with poly (dA:dT). Six hours post treatment, cells were discarded and native RIP assay was performed using anti-HUR1 antibody or antiHSP70 as negative control. Real-time PCR analysis for CXCL10, CCL5 (as negative control) and c-Myc (as positive control) was performed on the input (pull down) of the RIP samples, $n=3$ biologically independent experiments, SEM, one-way ANOVA followed by Dunnett's test, ( $\left.P f E V s-{ }^{\star} P=0.0427\right)$. NT (not treated). Source data are provided as a Source Data file. 
CXCL10 levels in malaria patients ${ }^{11,12}$ and is affected by the levels of host CXCL10 in malaria animal models of CM $4,6,7,13$. Thus, we investigated whether the released CXCL10 itself serves as a facilitating protein to modify parasitic growth. We suspected that at the early stages of the infection, the parasite actively reduces the secretion of CXCL10, but when the immune system is "out of control" (as in the severe disease case of CM) and the protein is heavily secreted, as detected in severe malaria patients ${ }^{4,6}$, the parasite can directly sense its presence and modify its growth.

To investigate this notion and to determine the effect of secreted CXCL10 on parasitic growth, we cultured a Pf NF54 strain under six different conditions, out of which, in three the CXCL10 protein was present and in the other three, absent. Briefly, we cultured the parasites under the following conditions: (1) RPMI fresh media; (2) THP-1 conditional media; (3) Conditional media supplemented with human recombinant CXCL10; (4) media harvested from THP-1 cells that were activated with $P f$-derived EVs lacking CXCL10 but containing other Type 1 IFN chemokines and cytokines to include CCL5, IFNa, and IFN $\beta$; (5) the same as in condition 4 but with supplemental CXCL10; and (6) media derived from THP-1 cells treated with Pf-DNA that constitutively secrete CXCL10, as well as other Type 1 IFN chemokines and cytokines.

Parasitemia levels were measured for 5 days (post two bloodstage cycles) using both flow cytometry (Fig. 5A) and Giemsa smears $^{63}$ (Fig. 5B, C). Remarkably, we observed the highest parasitemia levels using FACS counting (by $\sim 3$-fold) validated by Giemsa smear counting in the three conditions in which the parasites were exposed to CXCL10 as compared to conditions in which CXCL10 was absent but other chemokines were present (Fig. 5A, B). These results show that the presence of CXCL10 significantly increases parasite growth and imply that CXCL10 serves as a host protein that acts as a stimulator of parasitic growth dynamics.

We also investigated whether the increase in parasitemia, due to the presence of CXCL10, is a result of an increase in the number of daughter cells per schizont-infected cell. The parasites were cultured in the presence of CXC10 for $36 \mathrm{~h}$ and Giemsa smears were counted for the number of merozoites per a schizont-infected cell. We could not detect a statistically significant difference in the produced merozoite levels between the culture with and without the chemokine (Supplementary Figs. 22 and 23).

Further research is needed to understand the detailed sensing pathway responsible for the conversion of the CXCL10 signal into parasitic developmental readouts. Yet, to explore the effect of CXCL10 presence on the parasite, we monitored CXCL10 localization in trophozoite parasites treated with CXCL10 via immunofluorescence microscopy (IFA) (Supplementary Figs. 24 and 25). Parasite culture growing in the presence of the CCL5 chemokine served as control. Interestingly, immunofluorescence microscopy showed a strong CXCL10 signal within parasites treated with CXCL10, but neither CCL5 signal was detected in CCL5 treated parasites nor CXCL10 signal in not treated parasites (Supplementary Fig. 25).

Due to the autofluorescent nature of the parasite, we also monitored a few appropriate controls, to include primary antibody alone and secondary antibody alone. Microscopic monitoring showed a weak signal inside the parasite in all of the samples, however, in the CXCL10 treated cells the detected signal was significantly higher (Supplementary Fig. 25). Signal was not detected in uRBCs (Supplementary Fig. 24). These results suggest that CXCL10 might be accumulated inside the parasite. Western Blot analysis confirmed this observed signal of CXCL10 within infected cells, dematin was used as a host protein control
(Supplementary Fig. 26). These findings suggest that CXCL10 is transferred into the infected cell to stimulate a sensing pathway.

\section{Discussion}

During microbial infection, the dynamics of protein-secreted networks from the pathogen and host are constantly being used by both the host and pathogen to estimate and react as a means to ensure their respective development and survival. This crosstalk relies on a massive network of secreted proteins, including chemokines, from host cells and virulence effectors from the pathogens to ultimately determine the outcome of the infection. Thus, the presence of multiple host defense mechanisms drives pathogens to establish their own tools to sense the circumstances of the host and rewire their features.

In this study, we suggest the existence of an unexpected sensing pathway by which the elevation of the host chemokine CXCL10 levels triggers $P$. falciparum proliferation. Our findings point to a 'two-phase' model for disease progression (Fig. 6), which relies on a decision-sensing system that responds to CXCL10 levels: during early infection (or non-CM) malaria, when there is a tightly regulated immune response, the parasite modifies the immune response by inhibiting CXCL10 translation in monocytes (Fig. 6, upper panel), as supported by low CXCL10 levels in clinical samples of non-CM malaria patients ${ }^{11}$. Inhibiting CXCL10 translation can assist the parasite by, for instance, preventing a strong immunological response, by decreasing $\mathrm{T}$ cell activation. It can also assist the parasite with controlling the course of parasitemia as a mean to ensure its host survives (i.e., does not reach $\mathrm{CM}$ ) for the $\sim 2$ weeks it takes the gametocytes to sufficiently mature for transmission to the vector.

However, when the disease's circumstances become complicated and severe (as in CM), higher CXCL10 levels are detected in the blood circulation of patients ${ }^{4-6}$. Of note, although it appears that monocytes are one of the main sources of production of CXCL10 in response to $P$. falciparum infection ${ }^{64}$, this chemokine is released also by other cells such as neutrophils, eosinophils, and dendritic cells ${ }^{65-67}$. If and how the parasite communicates with these other host cells to alter CXCL10 production remains unknown.

Thus, this host-defense protein is, in fact, used by the parasite as a facilitator to evaluate how severe the host's situation is. When CXCL10 amounts reach a critical level, the 'alarm goes off, leading to Pf changes that promote its growth (Fig. 6, bottom panel) and, by these means, allow the parasite to avoid the detrimental consequences of the heightened immune response by escaping to the mosquito vector.

CXCL10 belongs to the family of chemokines, chemotactic cytokines, that play a double role:

(I) they function in host immune defense by orchestrating cellular movement during infection and (II) some also mediate the direct killing of various pathogens through a mechanism that is not fully understood ${ }^{68,69}$. CXCL10 has been previously shown to directly exert antimicrobial effects ${ }^{68}$ or to localize to the bacterial cell membrane to mediate direct microbial killing ${ }^{70}$. Yet, here we show that, in the case of malaria, instead of killing the parasite, CXCL10 is adopted by the parasite as facilitator to determine when to enhance growth.

Other pathogenic microbes are known to monitor host cytokine levels and thus change their gene expression in response. Neisseria meningitidis, for example, internalize TNF using their type IV pili, an interaction that leads to changes in their gene expression associated with hyper virulent phenotypes ${ }^{71}$. TNF likewise modulates Salmonella typhimurium virulent protein expression, including the effector SipA, which prompts increased bacterial invasion of human intestinal cells ${ }^{72}$. 
A.

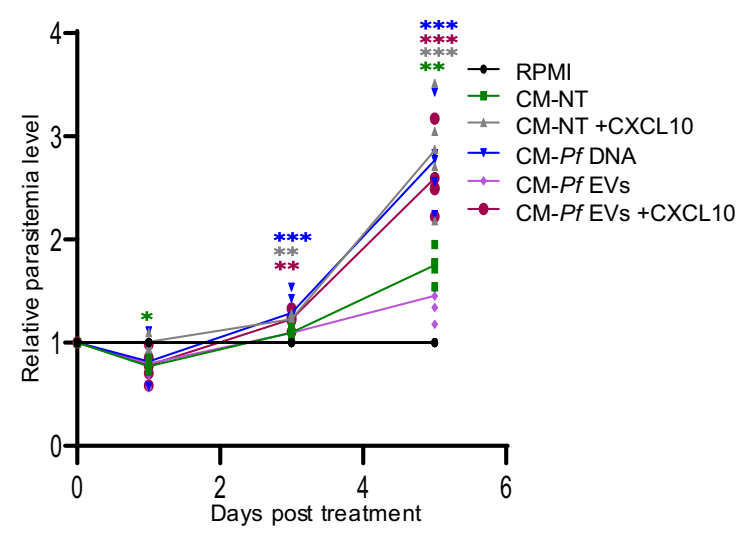

B.

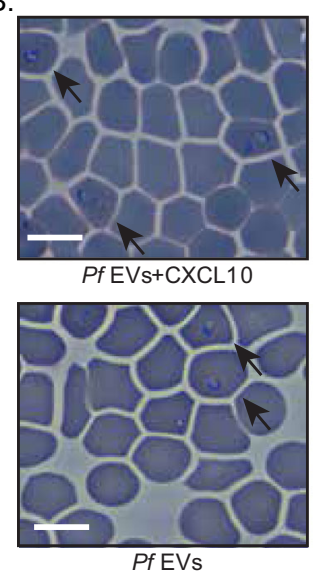

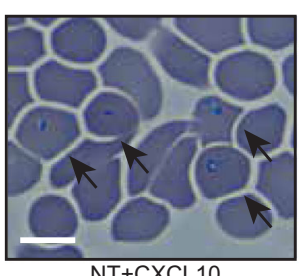

$\mathrm{NT}+\mathrm{CXCL} 10$

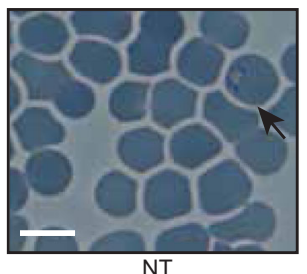

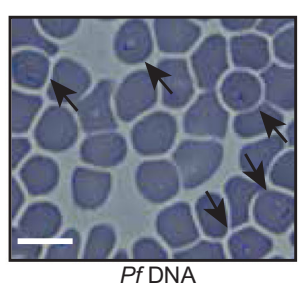

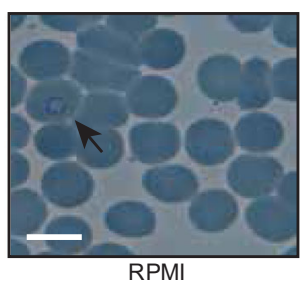

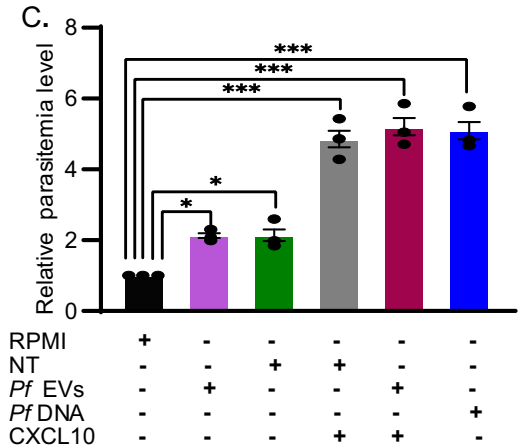

Fig. 5 The chemokine CXCL10 promotes parasite growth. A NF54 Pf parasites were cultured with media containing: (1) complete media [lacking CXCL10] (Black, RPMI); (2) media harvested from untreated THP-1 cells [lacking CXCL10] (Green, CM-NT); (3) media harvested from untreated THP-1 cells with supplemental $25 \mathrm{ng} / \mathrm{ml} \mathrm{CXCL10}$ [containing CXCL10] (gray, CM-NT+CXCL10); (4) media harvested from THP-1 cells pretreated with Pf-ring-stage-derived EVs [lacking CXCL10] (pink, CM-Pf-EVs); 5) Media harvested from THP-1 cells pretreated with Pf-ring-stage-derived EVs and supplemental $25 \mathrm{ng} / \mathrm{ml}$ CXCL10 [containing CXCL10] (red, CM-Pf EVs+CXCL10); or (6) Media harvested from THP-1 cells transfected with Pf gDNA [containing CXCL10] (blue, CM-Pf DNA). Parasitemia levels were measured every second day using flow cytometry. Graph shows relative parasitemia level. SEM, $n=5$ biologically independent experiments, one-way ANOVA followed by Dunnett's test, (1 day- CM-NT - RPMI ${ }^{\star} P=0.0435,3$ day- CM-NT + CXCL10 - RPMI ${ }^{\star \star} P=0.00480, C M-P f D N A-$ $\mathrm{RPMI}^{\star \star \star} P<0.001, \mathrm{CM}-\mathrm{Pf}$ EVs $+\mathrm{CXCL10}-\mathrm{RPMI}{ }^{\star \star} P=0.00489,5$ day- CM-NT - RPMI ${ }^{\star \star} P=0.00502, \mathrm{CM}-\mathrm{NT}+\mathrm{CXCL10}-\mathrm{RPMI}{ }^{\star \star \star} P<0.001, \mathrm{CM}-\mathrm{Pf} \mathrm{DNA}-$ RPMI ${ }^{\star \star \star} P<0.001, C M-P f E V s+C X C L 10-$ RPMI $\left.{ }^{\star \star \star} P<0.001\right)$. The data was analyzed using Diva v. 8.0.1 software (BD). The gating strategy is demonstrated in Supplementary Figure 27. B Representative Giemsa smears from a growth assay (A). Size bar $10 \mu \mathrm{m}$. Results are representative of at least three independent biological replicates. C Parasitemia counting using Giemsa smears from day 5 of the parasite culture $(A) . n=3$ biologically independent experiments, SEM, oneway ANOVA followed by Dunnett's test, (CM-NT - RPMI ${ }^{\star} P=0.0373$, CM-NT + CXCL10 - RPMI ${ }^{\star \star *} P<0.001$, CM-Pf DNA - RPMI ${ }^{\star \star *} P<0.001, C M-P f$ EVs RPMI ${ }^{*} P=0.0351, C M-P f E V s+C X C L 10-$ RPMI $\left.{ }^{\star \star *} P<0.001\right)$. Source data are provided as a Source Data file.

We here go beyond providing evidence that $P$. falciparum, while growing inside the RBC, can subvert the translation of CXCL10 in monocytes to identifying parasite and host elements involved in this mechanism: upon internalization of the RNA cargo, encapsulated in EVs, host RIG-I is activated, leading eventually to ribosome blockage on the CXCL10 transcript. Indeed, pathogens have established various sophisticated techniques, inducing translational inhibition, to govern their host's response. They modulate the immune response by targeting gene expression regulation (at the level of mRNA translation) via delivering effectors to the cytoplasm, which in time suppress host translation (reviewed in refs. ${ }^{73,}{ }^{74}$ ). The Leishmania parasite, for instance, promotes survival within macrophages by downregulating host-protein synthesis ${ }^{75}$; large DNA viruses such as adenoviruses or herpesviruses encode multiple effectors to prevent eIF2 $\alpha$ phosphorylation, resulting in host translation suppression $^{73}$. The Severe Acute Respiratory Virus (SARS) Coronavirus protein $\mathrm{Nsp} 1$ associates with the $40 \mathrm{~S}$ ribosomes to suppress host-gene expression by promoting host mRNA degradation ${ }^{76}$. Moreover, in a similar mechanism to the one presented in this study, infection by the Vesicular Stomatitis Virus leads to translation inhibition in the host ${ }^{38}$. This pathway depends on RIG-I-like receptors binding to the viral RNA, leading to subsequent interaction with STING, which consequently restricts the translation of diverse viral and host genes ${ }^{38}$.

From the host side, RIG-I receptors are known cytoplasmic key sensors of virus infection, mediating the transcriptional induction of the type I IFN pathway in order to establish an antiviral host innate and adaptive response. This receptor was shown to sense various RNA structures from a wide range of viruses (reviewed in Rehwinkel and $\mathrm{Gack}^{39}$ ). Nevertheless, it is now becoming clear that the role of RIG-I affects the immune cells in a much broader scope than just fighting viruses, for instance, the interaction with self-RNA (reviewed in Kato et al. ${ }^{77}$ ) and global translational initiation $^{38}$.

Our data indicate that the host RNA-binding protein HUR1 plays an important role in the inhibitory effect of $P f$-derived EV on CXCL10 translation. While in steady state conditions HUR1 is mostly confined to the nucleus, stress conditions can trigger HUR1 to move into the cytoplasm where it can complex specific target mRNAs by binding to AU-rich elements in the $3^{\prime} \mathrm{UTR}$. HUR1 is well known for its capacity to stabilize mRNAs, thereby enhancing their translation. However, we show that internalization of Pf-derived EVs into monocytes results in binding of 


\section{A. Early stage of malaria infection}

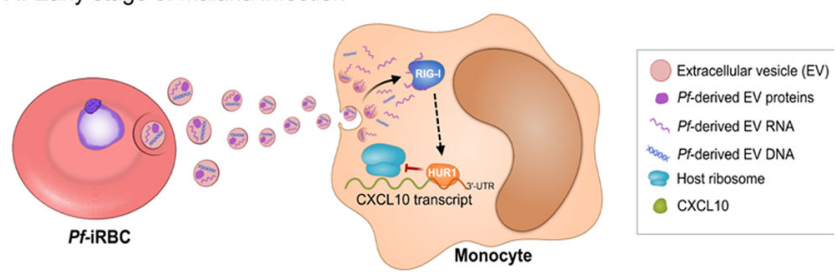

B. Late stage of malaria infection

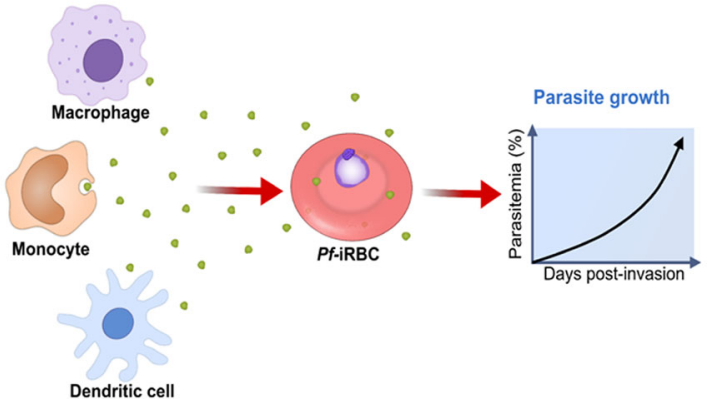

Fig. 6 proposed two-phase mechanism of CXCL10 regulation. A 'decision sensing system': A In non-complicated malaria (upper panel) the Pf-iRBCs repress CXCL10 expression in host monocytes via transferring of RNA cargo encapsulated in parasite-derived EVs. The RNA cargo is then recognized by the host RNA receptor, RIG-I, leading to a cascade of events that eventually blocks the association of the ribosomes to CXCL10 transcript in a process mediated by its 3'UTR and binding of HUR1. B However, in complicated malaria (such as CM) when CXCL10 level are higher, the chemokine is sensed back as an 'alert facilitator', prompting parasitic proliferation, possibly allowing an escape action (bottom panel). Other host immune cells produce and secrete CXCL10.

HUR1 to CXCL10 mRNA, and that the translation of CXCL10 is halted under these conditions. A potential explanation for this finding is the capacity of HUR1 to interact with negative modulators of translation. In myeloid cells it was shown that HUR1 stabilizes mRNAs for inflammatory mediators, but that HUR1 also synergizes with the translational silencer TIA-1 to reduce the translation of these mRNAs ${ }^{78}$. Via interactions with suppressive RBPs or microRNAs, HUR1 may therefore also act as a negative regulator of inflammation ${ }^{53,54,79}$. It remains to be determined which HUR1 interaction partners may be involved in $P f$-induced silencing of CXCL10 translation.

While our findings elucidate the mechanism that affects CXCL10 translation in monocytes, the mechanism by which $P f$ iRBCs sense the levels of CXCL10 in circulation and its downstream parasite targets are unknown and require further investigation. Several studies have shown that the malaria parasite imports host proteins to its cytosol, including human deltaaminolevulinate dehydratase ${ }^{80}$, ferrochelatase ${ }^{81}$, superoxide dismutase $^{82}$, and peroxiredoxin ${ }^{83}$. However, the exact mechanism by which $P f$-iRBCs transfer these proteins to the parasite cytoplasm is still poorly understood.

Other pathogens express proteins that specifically bind chemokines to better adapt to their hosts ${ }^{84}$. For instance, the human blood fluke Schistosoma mansoni secretes a chemokine-binding protein, SmCKBP, which attaches to CXCL8, CCL3, CX3CL1, CCL2, and CCL5, thereby blocking their interaction with their cell receptors ${ }^{85}$. Leishmania major expresses glycoprotein gp63, which cleaves CXCL10 and, consequently, impairs $\mathrm{T}$ cell chemotaxis $^{86}$. In the case of the dog tick Rhipicephalus sanguineus, this ectoparasite exports a family of glycoproteins, evasins, that show a high affinity to CXCL1 and CXCL8 and disrupt their binding to CXCR2, which ultimately inhibits CXCL8-mediated neutrophil migration ${ }^{87}$. Another example comes from the zoonotic Parapoxvirus orf (ORFV) which secretes a protein ${ }^{88}$ that binds to CCL19 and CCL21and inhibits the recruitment of immature and mature dendritic cells and $\mathrm{T}$ cell responses ${ }^{89}$.

We add another dimension to the functionality of the RIG-I receptor by demonstrating that its activation leads to a cascade of events that blocks the translation of CXCL10, a major chemokine in the anti-pathogenic immune response. Malaria parasites control this chemokine's expression in monocytes, re-designing the immune response by tricking the host's pattern recognition receptors (PRRs) for their own benefit, and in doing so, turning this 'host weapon' into a 'parasite weapon'.

\section{Methods}

Parasite Lines. NF54 was generously provided by the Malaria Research Reference Reagent Resource Center, MR4. NF54 Pf parasites were obtained through BEI Resources, NIAID, NIH: Plasmodium falciparum, Strain NF54 (Patient Line E), MRA-1000 was provided by Megan G. Dowler.

Parasite culture. $P f$ parasites were cultured in human red blood cells (RBCs) using standard methods ${ }^{90}$. Briefly, parasites were grown at $4 \%$ hematocrit in pooled uninfected RBCs, provided by the Israeli blood bank (Magen David Adom blood donations, Israel), and incubated at $37^{\circ} \mathrm{C}$ in a gas mixture of $1 \% \mathrm{O}_{2}$ and $5 \% \mathrm{CO}_{2}$ in $\mathrm{N}_{2}$. Parasites were maintained in RPMI medium $\mathrm{pH}$ 7.4, supplemented with $25 \mathrm{mg} / \mathrm{ml} \mathrm{HEPES}, 50 \mu \mathrm{g} / \mathrm{ml}$ hypoxanthine, $2 \mathrm{mg} / \mathrm{ml}$ sodium bicarbonate, $20 \mu \mathrm{g} / \mathrm{ml}$ gentamycin and $0.5 \%$ AlbumaxII. Pf cultures were tested once a month for the presence of Mycoplasma using MycoAlertPlus kits (Lonza). NF54 ring-stage P. falciparum parasites were synchronized using sorbitol lysis. Briefly, the media was removed from $P f$ cultures and the blood pellet was incubated at $37^{\circ} \mathrm{C}$ with $\mathrm{D}$ sorbitol (Sigma \#S1876) 5\% solution for $5 \mathrm{~min}$. Immediately after, sorbitol was removed and the blood pellet was washed twice with warm washing media. The blood pellet was re-suspended in complete media.

THP-1 cell culture. The monocytic cell line THP-1, commonly used in innate DNA sensing studies, was employed. Our EV uptake experiments were performed as previously described ${ }^{26,91}$ with $1 \mathrm{k} /$ per cell EVs were added to recipient cells. The RIG-I and MAVS KO THP-1 cell lines were generated by the CRISPR/Cas9 system (kindly provided by the laboratory of Professor Gunther Hartmann, University of Bonn, Germany). These cell lines were cultured as previously described ${ }^{92}$. The THP-1 culture was tested for the presence of Mycoplasma once a month using Hylabs Mycoplasma detection kit.

THP-1 KO lines. Gene-edited THP-1 cell lines were generated by transient electroporation with an EF1alpha-Cas9, U6-sgRNA ${ }^{93}$ expression plasmid targeting RIG-I (DDX58) (GGGTCTTCCGGATATAATCC(TGG)), MAVS

(GTCCTGCTCCTGATGCCCGC(TGG)), and $c G A S$

(GGCCGCCCGTCCGCGCAACT(GGG)), respectively. Gene-edited single cell clones were obtained by limiting dilutions and verified by Sanger sequencing and functional testing. Genotypes are listed in Table 1.

Purification of EVs from Pf-iRBC culture. EVs were harvested as described previously ${ }^{26}$. Briefly, $P f$-infected or uninfected RBC growth media was collected and cellular debris were removed by differential centrifugation at $400 \times g, 1,900 \times g$, and $17,000 \times g$. The supernatant was then concentrated using a Vivaflow 100,000 MWCO PES filter unit (Sartorious Stedium) and centrifuged at $150,000 \times g$ to pellet EVs. The EVs concentration was estimated using a Nanoparticle Ttracking Aanalysis (NTA) in a Nanosight NS300 instrument (Malvern Instruments, Worcestershire, UK) equipped with a $405-\mathrm{nm}$ laser and coupled to a CCD camera. Data was analyzed using NTA software (version 2.3).

Parasite growth assay. NF54 Pf parasites were synchronized by using 5\% sorbitol (D-sorbitol, S1876, Sigma) 1\% parasitemia level of ring stage NF54 Pf parasites $(\sim 4-8 \mathrm{~h}$ post invasion) were cultured in six-well plates, half the media was parasite RPMI complete media $(1.5 \mathrm{ml})$ and half derived from conditioned THP-1 culture: (i) naïve RPMI THP-1 media)RPMI((THP-1 complete media pre- THP-1 cells growth), (ii) THP-1 media from non-treated culture (CM-NT), (iii) THP-1 media from non-treated THP-1 culture supplemented with $25 \mathrm{ng} / \mathrm{ml}$ CXCL10 (CM-NT +CXCL10), (iv) THP-1 media from a culture pretreated with Pf-derived EVs (CMPf EVs) (the media was changed $1 \mathrm{~h}$ post EV incubation), (v) THP-1 media from a culture pretreated with $P f$-derived EVs supplemented (CM-Pf EVs+CXCL10)(the media was changed $1 \mathrm{~h}$ post EV incubation) with $25 \mathrm{ng} / \mathrm{ml} \mathrm{CXCL10,} \mathrm{or} \mathrm{(vi)} \mathrm{THP-1}$ 
Table 1 Sequence and insertion-deletion length of THP-1 knock-out genotypes for RIG-I, cGAS, and MAVS.

\begin{tabular}{lllll} 
Cell line & InDel allele $\mathbf{1}$ & Sequence allele $\mathbf{1}$ & Indel allele 2 & Sequence allele 2 \\
\hline THP-1 MAVS-/-\#1 & $-23 \mathrm{bp}$ & CCAGCG...CTGGG & Homozygous & - \\
THP-1 MAVS-/-\#2 & $1 \mathrm{bp}$ & CAGCGG.CATCAGG & Homozygous & - \\
THP-1 RIG-I (DDX58) & $-5 \mathrm{bp}$ & CCGGAT...CCTGGAAG & $-1 \mathrm{bp}$ & GATATA.TCCTGGA \\
$-/-\# 1$ & & & & \\
THP-1 RIG-I (DDX58) & $-4 \mathrm{bp}$ & GATATAA...GGAAGGC & $-5 \mathrm{bp}$ & ATATAAT...AAGGCTT \\
$-/-\# 2$ & & CCCCAGT.GCGCGGAC & $+1 \mathrm{bp}$ & CCCCAGT[+T]TGCGCGGAC \\
THP-1 CGAS-/-\#1 & $-1 \mathrm{bp}$ & CCCCAGT.GCGCGGAC & $-49 \mathrm{bp}$ & AGGCCG[+GCGGGTCTCGA CCCCCGTTCGCCTAGG]... \\
THP-1 CGAS-/- \#2 & $-1 \mathrm{bp}$ & & & GCGCCCC
\end{tabular}

media from a culture transfected with $P f$ gDNA (CM-Pf DNA). To monitor parasitemia levels, parasites were sampled every $48 \mathrm{~h}$ (at trophozoite stage) (blood cycle) and stained with $2 \mu \mathrm{M}$ Nuclear dye Hoechst 33342 (Invitrogen) and $0.01 \mu \mathrm{g} / \mathrm{ml} \mathrm{RNA} \mathrm{dye} \mathrm{Thiazole} \mathrm{Orange} \mathrm{(Sigma).} 30 \mathrm{~min}$ at $37^{\circ} \mathrm{C}$. Cells were washed once with PBS and analyzed using a BD Biosciences LSRII flow cytometer. Acquired data was analyzed with the Diva 8.0.1 software. The gating strategy is demonstrated in Supplementary Fig. 27.

Monocyte treatment with EVs. THP- 1 cell line was plated at a concentration of $1 \times 10^{6} \mathrm{cells} / 3 \mathrm{ml}$ media and treated with purified $P f$-derived, $T c$-derived or uRBCderived $\mathrm{EV}$ s at a final concentration of $1 \mathrm{k} \mathrm{EVs} / \mathrm{cell}$ for the time points specified in each experiment.

PBMC and primary monocyte isolation. Naïve peripheral blood mononuclear cells (PBMCs) were collected from three healthy donors (obtained from Israel's Magen David Adom naïve blood donor donations). CD14+ cells were isolated from the PBMCs by magnetic separation, using CD14 MicroBeads and an LS column (MACS, Miltenyi Biotec Inc.). One million CD14+ cells were plated per well and then incubated with $P f$-iRBC ring-stage-derived EVs. Monocytes were obtained by plating 3 million PBMCs in six-well plates for $1 \mathrm{~h}$ at $37^{\circ} \mathrm{C}$ with $5 \%$ $\mathrm{CO}_{2}$. Then, the cells were washed to remove non-adherent cells for a final $\sim 80 \%$ monocyte purity. Cells were grown in RPMI 1640 media supplemented with $10 \%$ FBS and $2 \mathrm{mM}$ L-glutamine.

EV RNA isolation. The EV pellet obtained from parasitic culture was washed with PBS and resuspended in Trizol -Tri-bio (Bio-Lab Ltd.) $)^{91}$. EV-RNA was extracted according to the manufacturer's Trizol protocol (Invitrogen). RNA concentration and purity were evaluated using spectrophotometrical NanoDrop quantification (NanoDrop 8000V2.3.2, Thermo Fisher Scientific, Massachusetts, USA).

Pf-nucleic acid production. Parasites were cultured in high parasitemia levels $(>5 \%)$ and tightly synchronized as described above. Ring- or trophozoite-stage parasite cultures were diluted 1:10 with a $0.2 \%$ saponin solution (Sigma) until all RBCs were lysed. Cells were washed with PBS $-/-$ at $3200 \times g$ for 4 min, until the supernatant was clear. For RNA isolation, cells were resuspended in $500 \mu \mathrm{l}$ Trizol (Bio-Lab Ltd.) according to the manufacturer's TRIzol protocol (Invitrogen). gDNA was extracted using the acid-phenol:chloroform procedure ${ }^{94}$ and subsequently sonicated to obtain $\sim 500$ bp sheared DNA sections.

Transfection into THP-1 cells. gDNA derived from the $P f$ NF54 strain or poly(dA: dT) ${ }^{95}$ were transfected into THP-1 cells using TransIT-X2 transfection reagent (Mirus Bio) according to the instructions of the manufacturer.

Transfection of RNA or $5^{\prime}$ triphosphate (InvivoGen Ltd.) into THP-1 cells was done using a Lipofectamine RNAiMAX Transfection Reagent (Thermo Fisher Scientific) according to the manufacturer's protocol.

Transfection of THP-1 cells with the following plasmids was performed using the Neon electroporation system (Thermo Fisher Scientific): CXCL10 3'-UTR (provided by GeneCopoeia Ltd.), HA-RIG-I plasmid (generously provided by the laboratory of Professor David Wallach from the Weizmann Institute of Science) and the three CXCL10 $3^{\prime} \mathrm{UTR}$ sub-domain $\Delta \mathrm{I}, \Delta \mathrm{II}$ and $\Delta \mathrm{III}$. The two deletions $\Delta \mathrm{II}$ and $\triangle \mathrm{III}$ were performed by Inverse PCR (SimpliAmp thermal cycler, Thermo Fischer), followed by ligation using the KLD kit, NEB, \#M0554. The deletion of the first segment $\Delta \mathrm{I}$ was done by using restriction enzyme (Ecor-I-Neb).

THP- 1 cells were washed with PBS at $37^{\circ} \mathrm{C}$ and the pellet was resuspended in a $100 \mu$ resuspension buffer $\mathrm{R}^{96}$. Cells were transfected with DNA under the following conditions using the Neon electroporation system: 1500 wavelength, 10 pulse width, 3 pulses (protocol provided by Prof. Andrew Bowie laboratory, Trinity College Dublin).

Validation of EV-derived RNA transfection of THP-1 cells. In order to validate the transfection of EV-RNA cargo into THP-1 cells, we isolated RNA from the cells an hour post transfection using the BioTri reagent and synthesized cDNA using the High Capacity cDNA Reverse Transcription kit (Applied Biosystems Ltd.). Then, PCR was conducted to detect the parasitic gene ETRAMP 11.2, using $200 \mathrm{ng}$ of cDNA as a template using primers listed in supplementary table 1 .

RNase digestion. EV RNA was treated with RNase H, RNase 1, or both (Thermo Fisher Scientific) according to the manufacturer's protocol to digest RNA-DNA hybrids or ssRNA, respectively.

Western blot assay. Pf-iRBC were saponized using $0.2 \%$ saponin in PBS (S7900Sigma) and then were lysed using RIPA buffer. EVs at a concentration of $1.5-2 \times$ $10^{11}$ particles per ml were lysed in $20 \mu \mathrm{l}$ of 5X RIPA buffer were sonicated in a bath sonicator for $10 \mathrm{~s}$. THP-1 cells were lysed using RIPA buffer. Then, $120 \mu \mathrm{g}$ of the obtained protein was subjected to gel electrophoresis; CXCL10 WB using 4-20\% Bis-Tris gradient gels (GeneScript) and transferred into PVDF membranes (Bio$\mathrm{Rad})$; the rest of WB using 10\% SDS-TRIS gels transferred into nitrocellulose membranes (Bio-Rad). The membrane was blocked for 1 hour with 5\% skim milk in PBS-Tween $200.05 \%$. The following primary antibodies were used in this work anti-CXCL10 produced in rabbit (Abcam) for $2 \mathrm{~h}$ or anti-HSP90 produced in mouse (Abcam) for 1 hour, both diluted 1:1000; Anti-HUR produced in rabbit (Santa Cruz), anti-GAPDH produced in rabbit (Abcam), and anti-Dematin produced in rabbit (Abcam) for $1 \mathrm{~h}$, all diluted 1:1000. The secondary antibodies used were goat anti-rabbit (Sigma-Aldrich) diluted 1:20,000 and goat anti-mouse (Abcam) diluted 1:2000, both conjugated with HRP and incubated for 40 minutes. The membrane was developed using Western ECL Substrate (Bio-Rad) and captured by Amersham imager 680 v2.0.0.

Measurement of cytokine production from monocytes. CXCL10 and CCL5 secreted from THP-1 cells were measured by ELISA according to the manufacturer's instructions (R\&D Systems). Type I IFN production was measured by a bioassay, using HEK-Blue ${ }^{m}$ IFN- $\alpha / \beta$ reporter cells (InvivoGen). Absorbance was measured by Tecan icontrol v. 3.9.1.0.

Quantitative real-time PCR. IFNB, IFNA, CCL5, and CXCL10 gene expression was determined by real-time PCR, using SYBR-fast green detection systems (ViiA 7 Real-Time PCR System, Applied Biosystems, QuantStudio real time PCR software v1.1). Expression levels were normalized to HTRP expression. Data for each phenotype is presented as the fold induction over untreated controls and represents the mean and standard deviation of at least three biological replicates and three technical replicates in each.

PCR primers used for this study are listed in Supplementary table 1.

Polysome profile assay. THP-1 cells transfected with $P f$-gDNA were treated with $P f$-derived EVs (in each biological replicate monocytes were treated with newly fresh purified EVs). Fourteen hours post treatment, ribosome profiling was conducted as previously described ${ }^{97}$. Briefly, THP-1 cells were grown in a $80-\mathrm{cm}^{2}$ flask, transfected with control poly(dA:dT), and then incubated with $P f$-derived EVs. Fourteen hours later, the cells were incubated with $100 \mu \mathrm{g} / \mathrm{ml}$ cycloheximide (CHX). Next, cells were lysed and used for ribosomal profiling and fractionation Then, RNA was isolated from heavy, light and free profile fractions. cDNA was then generated and gene expression was determined using RT-PCR as described above.

Flow cytometry for Pf. For flow cytometry analysis, cells were stained for $30 \mathrm{~min}$ at $37^{\circ} \mathrm{C}$ with staining solution containing $2 \mu \mathrm{M}$ Hoechst (Invitrogen, Waltham, Massachusetts, USA, cat\# H1399) and $10 \mathrm{ng} / \mathrm{mL}$ thiazole orange (Sigma-Aldrich, cat\# 390062) in PBS (Biological industries cat\#02-0230-1 A). Analysis was performed using LSRII flow cytometer equipped with a 355 and 488 laser and analyzed using DIVA 8.0.1 software. Gating for infected cells was based on uRBCs stained in the same conditions. 
Small interfering RNA assay. Dharmacon siGENOME Human SMARTpool siRNAs for HUR1, RIG-I or a non-relevant target (NR) were transfected into THP1 cells using Lipofectamine RNAiMAX Transfection Reagent (Thermo Fisher Scientific) according to the manufacturer's protocol. Protein expression was measured by western blot analysis $48 \mathrm{~h}$ post transfection.

RNA stability assay. THP-1 cells were treated with Pf-derived EVs or transfected with $P f$-gDNA. Twelve hours post treatment, $5 \mathrm{mg} / \mathrm{ml}$ actinomycin $\mathrm{D}$, or DMSO as control, was added to the cells for the indicated times. Cell samples were collected every $2 \mathrm{~h}$, from which the RNA was purified and cDNA synthesized as described above. CXCL10 and IFNB mRNA levels were assayed by quantitative RT-PCR, normalized to the housekeeping gene HPRT, and are presented as the remaining percentage compared to time 0 .

Immunofluorescence assay. Pf-ring stage iRBCs (5\% parasitemia) were synchronized and then cultured with $25 \mathrm{ng} / \mathrm{ml} \mathrm{CXCL10} \mathrm{( \# 300-12} \mathrm{PeproTech,} \mathrm{CCL5}$ (\#300-06 PeproTech) for $16 \mathrm{~h}$ or left untreated (NT). Pf-infected RBCs were fixed in a $4 \%$ paraformaldehyde and $0.0075 \%$ glutaraldehyde solution in PBS (Polysciences Inc.). Cells were permeabilized with $0.1 \%$ Triton X-10 in PBS for $10 \mathrm{~min}$ and then reduced with $0.1 \mathrm{mg} / \mathrm{ml} \mathrm{NaBH}$. Next, samples were blocked using $5 \%$ BSA for $30 \mathrm{~min}$, subsequently incubated overnight with primary antibodies: AntiCXCL10 (Abcam) and Anti CCL5/RANTES (A-4) (Santa Cruz) diluted 1:200, following by incubation for $1 \mathrm{~h}$ with a secondary goat anti-rabbit conjugated to Alexa Fluor 594 (life technologies) diluted 1:500 and Hoechst (molecular probs 33342). Fixed cells were spread on Poly-L coated cover slips using centrifuge spinning at $845 \times g$ for $15 \mathrm{~min}$. Cover slips were washed to get rid of unbound cells and mounted in VectaShield. All labeling experiments were conducted in parallel to controls, of primary antibody with omitted secondary antibody or secondary antibody only, in order to get the background staining and autofluorescence of the parasites. These controls were also quantified and showed very weak signal (see Supplementary Fig. 26).

Images were acquired using the DeltaVision microtiter system (Applied Precision, Inc., Issaquah, WA, USA), using a $\times 100 / 1.49$ oil objective (Olympus, Tokyo, Japan) or Eclipse TI-E Nikon inverted microscope, using Plan Apo $\times 100 /$ 1.4 NA. Image analysis was performed using ImageJ software (rsbweb.nih.gov/ij, ImageJ $1.51 \mathrm{k}, \mathrm{R}$ v.4). In order to avoid sample to sample variation the mean intensity inside multiple parasites in each sample was quantified $(7<n<23)$. Parasites were chosen unbiasedly, based on the brightfield images. Background values were measured as well for each sample and subtracted from the intensity values (relative grayscale values).

Luciferase assay. The CXCL10-3'UTR-Luciferase system (GeneCopoeia Ltd.) was used to determine the CXCL10 translation rate. The secreted levels of luciferase and the normalizer SEAP were measured by a plate reader (TECAN INFINITE M PLEX, Tecan icontrol v. 3.9.1.0) using the Secrete-Pair Dual Luminescence Assay Kit (GeneCopoeia) according to the instructions of the manufacturer.

Trypanosoma cruzi EV purification. T. cruzi Y strain (EV-TY) EVs were purified by size-exclusion chromatography, using a Sepharose CL-4B column $(1 \times 40 \mathrm{~cm}$, GE Healthcare Systems, NJ, USA) as previously described ${ }^{98}$. NTA analysis (NTA v. 2.3 (Nanosight)) was performed to estimate the concentration and size of the EVs, as described above.

Protein degradation assay. THP- 1 cells were treated with the proteasome inhibitor MG132 in parallel to treatment with Pf-derived EVs or transfected with poly $(\mathrm{dA}: \mathrm{dT})$. Fourteen hours later, cells were collected and protein levels were analyzed by immunoblotting.

RNA maturation assay. THP-1 cells were treated with Pf-derived EVs and then incubated for 12 hours. In each biological replicate monocytes were treated with newly fresh purified EVs. Next, the RNA was isolated and cDNA was synthesized. PCR analysis was performed for the mature and immature CXCL10 species and detected with ethidium bromide-stained agarose gels.

RNA immunoprecipitation. Native RNA immune precipitation (RIP) was performed following a published protocol ${ }^{99}$. The Immunoprecipitation anti-HUR1 was performed with $5 \mu \mathrm{g}$ anti-HUR1 (Santa Cruz, 3a2, SC-5261), anti-AUF1 (Santa Cruz sc-166577), and anti-HSP70 (Santa Cruz, k-20, sc-1060) antibodies per sample.

Statistical analysis. Experiments were performed independently and at least in three biological replicates. The data are shown as the mean \pm standard error (SEM). One-way ANOVA followed by Dunnett's test were used to perform statistical

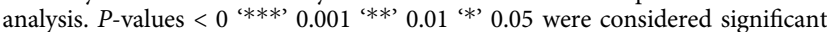
Differences in $\$ 4$ were tested with a mixed effects model, with treatment and time as fixed factors, and replicate (flask) as a random factor. All statistical tests were run in R, v. 4.0.3 and Prism 9. Mixed models were created using the R package 'ImerTest', v. 3.1.

Reporting summary. Further information on research design is available in the Nature Research Reporting Summary linked to this article.

\section{Data availability}

The datasets generated during and/or analyzed during the current study are available from the corresponding author on reasonable request. Source data are provided with this paper.

Received: 30 July 2020; Accepted: 14 July 2021; Published online: 11 August 2021

\section{References}

1. Rieke, B. Overview of the worldwide Handling with the Disease in Prevention, Diagnostics and Therapy WHO World Malaria Report 2017. Flugmed. Tropenme 25, 5-5 (2018)

2. Verlinden, B. K., Louw, A. \& Birkholtz, L. M. Resisting resistance: is there a solution for malaria? Expert Opin. Drug Discov. 11, 395-406 (2016).

3. Dunst, J., Kamena, F. \& Matuschewski, K. Cytokines and chemokines in cerebral malaria pathogenesis. Front. Cell. Infect. Microbiol 7, 324 (2017).

4. Jain, V. et al. Plasma IP-10, apoptotic and angiogenic factors associated with fatal cerebral malaria in India. Malar. J. 7, 83 (2008).

5. Wilson, N. O. et al. CXCL4 and CXCL10 predict risk of fatal cerebral malaria. Dis. Markers 30, 39-49 (2011).

6. Armah, H. B. et al. Cerebrospinal fluid and serum biomarkers of cerebral malaria mortality in Ghanaian children. Malar. J. 6, 147 (2007).

7. Ioannidis, L. J. et al. Monocyte- and neutrophil-derived CXCL10 impairs efficient control of blood-stage malaria infection and promotes severe disease J. Immunol. 196, 1227-1238 (2016)

8. Wassmer, S. C. \& Grau, G. E. Severe malaria: what's new on the pathogenesis front? Int. J. Parasitol. 47, 145-152 (2017).

9. Vazirinejad, R., Ahmadi, Z., Kazemi Arababadi, M., Hassanshahi, G. \& Kennedy, D. The biological functions, structure and sources of CXCL10 and its outstanding part in the pathophysiology of multiple sclerosis. Neuroimmunomodulation 21, 322-330 (2014).

10. Ahmadi, Z., Arababadi, M. K. \& Hassanshahi, G. CXCL10 activities, biological structure, and source along with its significant role played in pathophysiology of type I diabetes mellitus. Inflammation 36, 364-371 (2013).

11. Aguilar, R. et al. Changing plasma cytokine, chemokine and growth factor profiles upon differing malaria transmission intensities. Malar. J. 18, 406 (2019).

12. Georgiadou, A. et al. Modelling pathogen load dynamics to elucidate mechanistic determinants of host-Plasmodium falciparum interactions. Nat. Microbiol. 4, 1592-1602 (2019).

13. Nie, C. Q. et al. IP-10-mediated T cell homing promotes cerebral inflammation over splenic immunity to malaria infection. PLoS Pathog. 5 e1000369 (2009).

14. Tadesse, F. G. et al. Gametocyte sex ratio: the key to understanding plasmodium falciparum transmission? Trends Parasitol. 35, 226-238 (2019).

15. Ofir-Birin, Y. \& Regev-Rudzki, N. Extracellular vesicles in parasite survival. Science 363, 817-818 (2019).

16. Hosseini-Beheshti, E. \& Grau, G. E. R. Extracellular vesicles as mediators of immunopathology in infectious diseases. Immunol. Cell Biol. https://doi.org/ 10.1111/imcb.12044 (2018).

17. Antwi-Baffour, S. et al. Plasma mEV levels in Ghanain malaria patients with low parasitaemia are higher than those of healthy controls, raising the potential for parasite markers in $\mathrm{mEVs}$ as diagnostic targets. J. Extracell. Vesicles 9, 1697124 (2020).

18. Toda, H. et al. Plasma-derived extracellular vesicles from Plasmodium vivax patients signal spleen fibroblasts via NF-kB facilitating parasite cytoadherence. Nat. Commun. 11, 2761 (2020).

19. Chavez, A. S. O., O’Neal, A. J., Santambrogio, L., Kotsyfakis, M. \& Pedra, J. H. F. Message in a vesicle - trans-kingdom intercommunication at the vector-host interface. J. Cell Sci. https://doi.org/10.1242/jcs.224212 (2019).

20. Schorey, J. S., Cheng, Y., Singh, P. P. \& Smith, V. L. Exosomes and other extracellular vesicles in host-pathogen interactions. EMBO Rep. 16, 24-43 (2015).

21. Dong, G., Filho, A. L. \& Olivier, M. Modulation of host-pathogen communication by extracellular vesicles (EVs) of the protozoan parasite leishmania. Front. Cell. Infect. Microbiol. 9, 100 (2019).

22. van Dongen, H. M., Masoumi, N., Witwer, K. W. \& Pegtel, D. M. Extracellular vesicles exploit viral entry routes for cargo delivery. Microbiol. Mol. Biol. Rev. 80, 369-386 (2016) 
23. Regev-Rudzki, N. et al. Cell-cell communication between malaria-infected red blood cells via exosome-like vesicles. Cell 153, 1120-1133 (2013).

24. Mantel, P. Y. et al. Malaria-infected erythrocyte-derived microvesicles mediate cellular communication within the parasite population and with the host immune system. Cell Host Microbe 13, 521-534 (2013).

25. Mantel, P. Y. et al. Infected erythrocyte-derived extracellular vesicles alter vascular function via regulatory Ago2-miRNA complexes in malaria. Nat. Commun. 7, 12727 (2016).

26. Sisquella, X. et al. Malaria parasite DNA-harbouring vesicles activate cytosolic immune sensors. Nat. Commun. 8, 1985 (2017).

27. Gualdron-Lopez, M. et al. Characterization of plasmodium vivax proteins in plasma-derived exosomes from malaria-infected liver-chimeric humanized mice. Front. Microbiol. 9, 1271 (2018).

28. Demarta-Gatsi, C. et al. Histamine releasing factor and elongation factor 1 alpha secreted via malaria parasites extracellular vesicles promote immune evasion by inhibiting specific T cell responses. Cell Microbiol. 21, e13021 (2019).

29. Debs, S. et al. Interplay of extracellular vesicles and other players in cerebral malaria pathogenesis. Biochim. Biophys. Acta Gen. Subj. 1863, 325-331 (2019).

30. Couper, K. N. et al. Parasite-derived plasma microparticles contribute significantly to malaria infection-induced inflammation through potent macrophage stimulation. PLoS Pathog. 6, e1000744 (2010).

31. El-Assaad, F., Wheway, J., Hunt, N. H., Grau, G. E. \& Combes, V. Production, fate and pathogenicity of plasma microparticles in murine cerebral malaria. PLoS Pathog. 10, e1003839 (2014).

32. Schlee, M. \& Hartmann, G. Discriminating self from non-self in nucleic acid sensing. Nat. Rev. Immunol. 16, 566-580 (2016).

33. Cornejo, E., Schlaermann, P. \& Mukherjee, S. How to rewire the host cell: a home improvement guide for intracellular bacteria. J. Cell Biol. 216, 3931-3948 (2017).

34. Moscovitz, O. et al. The Parkinson's-associated protein DJ-1 regulates the $20 \mathrm{~S}$ proteasome. Nat. Commun. 6, 6609 (2015)

35. Garland, W. \& Jensen, T. H. Nuclear sorting of RNA. Wiley Interdiscip. Rev. RNA 11, e1572 (2020)

36. Maess, M. B., Wittig, B. \& Lorkowski, S. Highly efficient transfection of human THP-1 macrophages by nucleofection. J. Vis. Exp. e51960, https://doi. org/10.3791/51960 (2014).

37. Chan, Y. K. \& Gack, M. U. Viral evasion of intracellular DNA and RNA sensing. Nat. Rev. Microbiol. 14, 360-373 (2016).

38. Franz, K. M., Neidermyer, W. J., Tan, Y. J., Whelan, S. P. J. \& Kagan, J. C. STING-dependent translation inhibition restricts RNA virus replication. Proc. Natl Acad. Sci. USA 115, E2058-E2067 (2018).

39. Rehwinkel, J. \& Gack, M. U. RIG-I-like receptors: their regulation and roles in RNA sensing. Nat. Rev. Immunol. https://doi.org/10.1038/s41577-020-0288-3 (2020).

40. Hornung, V. et al. 5'-Triphosphate RNA is the ligand for RIG-I. Science 314, 994-997 (2006).

41. Rebane, A. \& Akdis, C. A. MicroRNAs: essential players in the regulation of inflammation. J. Allergy Clin. Immunol. 132, 15-26 (2013).

42. Szostak, E. \& Gebauer, F. Translational control by 3'-UTR-binding proteins. Brief. Funct. Genomics 12, 58-65 (2013).

43. Treiber, T., Treiber, N. \& Meister, G. Regulation of microRNA biogenesis and function. Thromb. Haemost. 107, 605-610 (2012).

44. Freen-van Heeren, J. J., Popovic, B., Guislain, A. \& Wolkers, M. C. Human $\mathrm{T}$ cells employ conserved AU-rich elements to fine-tune IFN-gamma production. Eur. J. Immunol. https://doi.org/10.1002/eji.201948458 (2020).

45. Rataj, F. et al. Targeting AU-rich element-mediated mRNA decay with a truncated active form of the zinc-finger protein TIS11b/BRF1 impairs major hallmarks of mammary tumorigenesis. Oncogene 38, 5174-5190 (2019).

46. Vlasova-St Louis, I. \& Bohjanen, P. R. Post-transcriptional regulation of cytokine signaling by AU-rich and GU-rich elements. J. Interferon Cytokine Res. 34, 233-241 (2014).

47. Tebo, J. et al. Heterogeneity in control of mRNA stability by AU-rich elements. J. Biol. Chem. 278, 12085-12093 (2003).

48. Bakheet, T., Hitti, E. \& Khabar, K. S. A. ARED-Plus: an updated and expanded database of AU-rich element-containing mRNAs and pre-mRNAs. Nucleic Acids Res. 46, D218-D220 (2018).

49. Jiang, Q. et al. Hyper-editing of cell-cycle regulatory and tumor suppressor RNA promotes malignant progenitor propagation. Cancer Cell 35, 81-94.e87 (2019).

50. Bakheet, T., Hitti, E., Al-Saif, M., Moghrabi, W. N. \& Khabar, K. S. A. The AU-rich element landscape across human transcriptome reveals a large proportion in introns and regulation by ELAVL1/HuR. Biochim. Biophys. Acta Gene Regul. Mech. 1861, 167-177 (2018).

51. Lu, Y. C. et al. ELAVL1 modulates transcriptome-wide miRNA binding in murine macrophages. Cell Rep. 9, 2330-2343 (2014).
52. Terasaka, T. et al. The RNA-binding protein ELAVL1 regulates GnRH receptor expression and the response to GnRH. Endocrinology 160, 1999-2014 (2019).

53. Yiakouvaki, A. et al. Myeloid cell expression of the RNA-binding protein HuR protects mice from pathologic inflammation and colorectal carcinogenesis. J. Clin. Invest. 122, 48-61 (2012).

54. Christodoulou-Vafeiadou, E. et al. Divergent innate and epithelial functions of the RNA-binding protein HuR in intestinal inflammation. Front. Immunol. 9, 2732 (2018)

55. Lafon, I., Carballes, F., Brewer, G., Poiret, M. \& Morello, D. Developmental expression of AUF1 and HuR, two c-myc mRNA binding proteins. Oncogene 16, 3413-3421 (1998).

56. Rhee, W. J. et al. HuR regulates the expression of stress-sensitive genes and mediates inflammatory response in human umbilical vein endothelial cells Proc. Natl Acad. Sci. USA 107, 6858-6863 (2010).

57. Fan, J. et al. Chemokine transcripts as targets of the RNA-binding protein HuR in human airway epithelium. J. Immunol. 186, 2482-2494 (2011).

58. Sedlyarov, V. et al. Tristetraprolin binding site atlas in the macrophage transcriptome reveals a switch for inflammation resolution. Mol. Syst. Biol. 12, 868 (2016).

59. Kim, H. H. et al. HuR recruits let-7/RISC to repress c-Myc expression. Genes Dev. 23, 1743-1748 (2009).

60. Zhang, W. et al. Purification, characterization, and cDNA cloning of an AUrich element RNA-binding protein, AUF1. Mol. Cell Biol. 13, 7652-7665 (1993).

61. Abbadi, D., Yang, M., Chenette, D. M., Andrews, J. J. \& Schneider, R. J. Muscle development and regeneration controlled by AUF1-mediated stage-specific degradation of fate-determining checkpoint mRNAs. Proc. Natl Acad. Sci. USA 116, 11285-11290 (2019).

62. Stone, M. J., Hayward, J. A., Huang, C. Z. E. H. \& Sanchez, J. Mechanisms of regulation of the chemokine-receptor network. Int. J. Mol. Sci. https://doi.org/ 10.3390/ijms18020342 (2017).

63. Dekel, E. et al. Identification and classification of the malaria parasite blood developmental stages, using imaging flow cytometry. Methods 112, 157-166 (2017).

64. Ioannidis, L. J., Eriksson, E. \& Hansen, D. S. CD14(+) monocytes are the main leucocytic sources of CXCL10 in response to Plasmodium falciparum. Parasitology 147, 465-470 (2020).

65. Dyer, K. D., Percopo, C. M., Fischer, E. R., Gabryszewski, S. J. \& Rosenberg, H F. Pneumoviruses infect eosinophils and elicit MyD88-dependent release of chemoattractant cytokines and interleukin-6. Blood 114, 2649-2656 (2009).

66. Liu, M. et al. CXCL10/IP-10 in infectious diseases pathogenesis and potential therapeutic implications. Cytokine Growth Factor Rev. 22, 121-130 (2011).

67. Luster, A. D. \& Ravetch, J. V. Biochemical characterization of a gamma interferon-inducible cytokine (IP-10). J. Exp. Med. 166, 1084-1097 (1987).

68. Crawford, M. A. et al. Antimicrobial effects of interferon-inducible CXC chemokines against Bacillus anthracis spores and bacilli. Infect. Immun. 77, 1664-1678 (2009)

69. Yang, D. et al. Many chemokines including CCL20/MIP-3alpha display antimicrobial activity. J. Leukoc. Biol. 74, 448-455 (2003).

70. Crawford, M. A. et al. Identification of the bacterial protein FtsX as a unique target of chemokine-mediated antimicrobial activity against Bacillus anthracis. Proc. Natl Acad. Sci. USA 108, 17159-17164 (2011).

71. Mahdavi, J. et al. Pro-inflammatory cytokines can act as intracellular modulators of commensal bacterial virulence. Open Biol. 3, 130048 (2013).

72. Ma, J., Zhang, Y. G., Xia, Y. \& Sun, J. The inflammatory cytokine tumor necrosis factor modulates the expression of Salmonella typhimurium effector proteins. J. Inflamm. 7, 42 (2010).

73. Mohr, I. \& Sonenberg, N. Host translation at the nexus of infection and immunity. Cell Host Microbe 12, 470-483 (2012).

74. Troemel, E. Host detection of pathogen-induced translational inhibition: a new pathogen-specific branch of the innate immune system? Future Microbiol. 7, 1133-1136 (2012).

75. Jaramillo, M. et al. Leishmania repression of host translation through mTOR cleavage is required for parasite survival and infection. Cell Host Microbe $\mathbf{9}$, 331-341 (2011).

76. Kamitani, W. et al. Severe acute respiratory syndrome coronavirus nsp1 protein suppresses host gene expression by promoting host mRNA degradation. Proc. Natl Acad. Sci. USA 103, 12885-12890 (2006).

77. Kato, H., Oh, S. W. \& Fujita, T. RIG-I-like receptors and type I interferonopathies. J. Interferon Cytokine Res. 37, 207-213 (2017)

78. Katsanou, V. et al. The RNA-binding protein Elavl1/HuR is essential for placental branching morphogenesis and embryonic development. Mol. Cell Biol. 29, 2762-2776 (2009).

79. Kawai, T. et al. Translational control of cytochrome $c$ by RNA-binding proteins TIA-1 and HuR. Mol. Cell Biol. 26, 3295-3307 (2006). 
80. Bonday, Z. Q., Dhanasekaran, S., Rangarajan, P. N. \& Padmanaban, G. Import of host delta-aminolevulinate dehydratase into the malarial parasite: identification of a new drug target. Nat. Med. 6, 898-903 (2000).

81. Varadharajan, S., Sagar, B. K., Rangarajan, P. N. \& Padmanaban, G. Localization of ferrochelatase in Plasmodium falciparum. Biochem. J. 384, 429-436 (2004).

82. Fairfield, A. S., Meshnick, S. R. \& Eaton, J. W. Malaria parasites adopt host cell superoxide dismutase. Science 221, 764-766 (1983).

83. Koncarevic, S. et al. The malarial parasite Plasmodium falciparum imports the human protein peroxiredoxin 2 for peroxide detoxification. Proc. Natl Acad. Sci. USA 106, 13323-13328 (2009).

84. Stone, M. J., Hayward, J. A., Huang, C., Huma, Z. E. \& Sanchez, J. Mechanisms of regulation of the chemokine-receptor network. Int. J. Mol. Sci. 18, 342 (2017).

85. Smith, P. et al. Schistosoma mansoni secretes a chemokine binding protein with antiinflammatory activity. J. Exp. Med. 202, 1319-1325 (2005).

86. Antonia, A. L. et al. Pathogen evasion of chemokine response through suppression of CXCL10. Front. Cell. Infect. Microbiol. 9, 280 (2019).

87. Denisov, S. S. et al. Tick saliva protein Evasin-3 modulates chemotaxis by disrupting CXCL8 interactions with glycosaminoglycans and CXCR2. J. Biol. Chem. 294, 12370-12379 (2019).

88. Seet, B. T. et al. Analysis of an orf virus chemokine-binding protein: Shifting ligand specificities among a family of poxvirus viroceptors. Proc. Natl Acad. Sci. USA 100, 15137-15142 (2003).

89. Lateef, $Z$. et al. The chemokine-binding protein encoded by the poxvirus orf virus inhibits recruitment of dendritic cells to sites of skin inflammation and migration to peripheral lymph nodes. Cell Microbiol. 12, 665-676 (2010).

90. Trager, W. \& Jensen, J. B. Human malaria parasites in continuous culture. Science 193, 673-675 (1976).

91. Ofir-Birin, Y. et al. Monitoring extracellular vesicle cargo active uptake by imaging flow cytometry. Front. Immunol. 9, 1011 (2018).

92. Unterholzner, L. et al. IFI16 is an innate immune sensor for intracellular DNA. Nat. Immunol. 11, 997-1004 (2010).

93. Mali, P. et al. RNA-guided human genome engineering via Cas9. Science 339, 823-826 (2013).

94. Triglia, T. \& Cowman, A. F. Primary structure and expression of the dihydropteroate synthetase gene of Plasmodium falciparum. Proc. Natl Acad. Sci. USA 91, 7149-7153 (1994).

95. Ma, F. et al. Positive feedback regulation of type I IFN production by the IFNinducible DNA sensor cGAS. J. Immunol. 194, 1545-1554 (2015).

96. Musilova, J., Mulcahy, M. E., Kuijk, M. M., McLoughlin, R. M. \& Bowie, A. G. Toll-like receptor 2-dependent endosomal signaling by Staphylococcus aureus in monocytes induces type I interferon and promotes intracellular survival. $J$. Biol. Chem. 294, 17031-17042 (2019).

97. Ingolia, N. T. Ribosome footprint profiling of translation throughout the genome. Cell 165, 22-33 (2016).

98. Torrecilhas, A. C. T. et al. Trypanosoma cruzi: parasite shed vesicles increase heart parasitism and generate an intense inflammatory response. Microbes Infect. 11, 29-39 (2009).

99. Gagliardi, M. \& Matarazzo, M. R. RIP: RNA Immunoprecipitation. Methods Mol. Biol. 1480, 73-86 (2016).

\section{Acknowledgements}

We thank the Malaria Research Reference Reagent Resource Center (MR4) for their generous supply of parasite strains. We thank Dr Silvia Haase and Dr Tom Driedonks for the fruitful scientific discussions. ENMNtH was supported by the European Research Council under the European Union's Seventh Framework Programme [FP/2007-2013] / ERC Grant Agreement number [337581]. The research of NR-R is supported by the
Benoziyo Endowment Fund for the Advancement of Science, the Jeanne and Joseph Nissim Foundation for Life Sciences Research and the Samuel M. Soref and Helene K. Soref Foundation. NR-R is supported by a research grant from David E. and Sheri Stone and by a research grant from Richard and Mica Hadar. N.R-R. is the incumbent of the Enid Barden and Aaron J. Jade President's Development Chair for New Scientists in Memory of Cantor John Y. Jade. N.R-R. is grateful for the support from the European Research Council (ERC) under the European Union's Horizon 2020 research and innovation program (grant agreement No. 757743), the Israel Science Foundation (ISF) (619/16 and 2235/16), the Israel Precision Medicine Program (IPMP) Research Grant Application no. 1637/20, and the Weizmann - Sao Paulo Research Foundation (FAPESP) Brazil, supported by a research grant from the Instituto Serrapilheira.

\section{Author contributions}

Y.O.B., H.B.A.P., and A.C.C. designed and performed the experiments, data analysis, and figure and manuscript preparation. Ar.R., An.R., N.R., T.B.T., Y.P., P.A.K., E.K., A.S., and T.H.S. assisted in parasite culture work, molecular biochemical and immune assays. R.R. assisted in statistical analysis. A.C.T. performed Trypanosoma cruzi-EV experiment. O.Y.R. and R.N. performed IFA experiment and imaging data analysis. Z.P. assisted in FACS analysis. Al.R. created the summarizing model. A.D.P. assisted in bioinformatic analysis. T.Z. and G.H. provided Crispr/cas9 KO lines. E.N.M.N.H. provided key scientific insights on translation regulators. D.A., E.S., N.B.Q., R.D., M.G., C.L., Al.R., and A.G.B. interpreted the data, provided scientific input, and contributed to the data presentation. N.R.R. designed the experiments wrote the manuscript with input from coauthors.

\section{Competing interests}

The authors declare no competing interests

\section{Additional information}

Supplementary information The online version contains supplementary material available at https://doi.org/10.1038/s41467-021-24997-7.

Correspondence and requests for materials should be addressed to N.R-R.

Peer review information Nature Communications thanks Serena Baglio, Daniel Fernandez-Ruiz, and the other, anonymous reviewer(s) for their contribution to the peer review of this work.

Reprints and permission information is available at http://www.nature.com/reprints

Publisher's note Springer Nature remains neutral with regard to jurisdictional claims in published maps and institutional affiliations.

pen Access This article is licensed under a Creative Commons Attribution 4.0 International License, which permits use, sharing, adaptation, distribution and reproduction in any medium or format, as long as you give appropriate credit to the original author(s) and the source, provide a link to the Creative Commons license, and indicate if changes were made. The images or other third party material in this article are included in the article's Creative Commons license, unless indicated otherwise in a credit line to the material. If material is not included in the article's Creative Commons license and your intended use is not permitted by statutory regulation or exceeds the permitted use, you will need to obtain permission directly from the copyright holder. To view a copy of this license, visit http://creativecommons.org/ licenses/by/4.0/.

(C) The Author(s) 2021 